\title{
Differential gene expression analysis and weighted gene co-expression network analysis reveal mechanism behind the development of duodenal carcinoma in familial adenomatous polyposis
}

\author{
Wei-Qing Liu \\ Kunming Medical University First Affilliated Hospital \\ Xuan-Yu Meng \\ Kunming Medical University First Affilliated Hospital \\ Xiao-Ting Liu \\ Kunming Medical University First Affilliated Hospital \\ Lei Liang \\ Kunming Medical University First Affilliated Hospital \\ Zhi-Yong Kou \\ Kunming Medical University First Affilliated Hospital \\ Wen-Liang Li \\ Kunming Medical University First Affilliated Hospital \\ Jun Yang ( $\square$ yangjun6@kmmu.edu.cn )
}

Kunming Medical University First Affilliated Hospital https://orcid.org/0000-0002-8100-6943

\section{Primary research}

Keywords: familial adenomatous polyposis, duodenal carcinoma, mechanism, bioinformatics analysis, immunotherapy

Posted Date: February 24th, 2021

DOI: https://doi.org/10.21203/rs.3.rs-218456/v1

License: (c) (1) This work is licensed under a Creative Commons Attribution 4.0 International License. Read Full License 


\section{Abstract \\ Background}

Duodenal carcinoma is the third cause of mortality in familial adenomatous polyposis (FAP) patients. The molecular mechanism by which FAP triggers and regulates duodenal carcinoma development was seldom studied so far.

\section{Objective}

The present study sought to use the bioinformatics approaches to provide novel insights into the molecular mechanism of FAP developing into duodenal carcinoma.

\section{Methods}

Based on GSE111156 dataset, differential expression analysis and weighted gene co-expression network analysis (WGCNA) were performed to identify differentially expressed genes (DEGs) and key gene modules, respectively. The functional enrichment analysis was conducted R package "clusterProfiler" and biological functions and pathways related to the immune system were further identified.

\section{Results}

Between adenoma tissue samples from FAP patients with and without duodenal carcinoma, 13 upregulated genes including MIR4747, THBS1, and RNU6_62 and 113 down-regulated genes including AKR1B10, AGAP9 and AKR1C3 were identified. These DEGs were mainly involved in terpnoid metabolism. In WGCNA analysis, the blue module associated with duodenal carcinoma in FAP contained 5393 genes including 11 hub genes and was mainly related to the regulation of neuron projection development. In tissues from FAP patients with duodenal carcinoma, 32 up-regulated genes including INHBA, COL3A1, and COL1A1 and 18 down-regulated genes including MT1H, KIAA1324, and HMGCS2 were screened between adenocarcinoma and normal tissues and were also significantly related to terpnoid metabolism. Between adenocarcinoma and adenoma tissues, we screened 171 up-regulated genes including CEACAM5, SLC2A1 and PKMRNU6_62 and 238 downregulated genes including RBP2, GSTA5 and SST, which were mainly involved in extracellular matrix organization. WGCNA revealed that the darkorange module associated with adenocarcinoma contained 554 genes including 19 hub genes and was involved in extracellular matrix organization and focal adhesion. Further identification of immune related processes showed that leukocyte migration was the process mostly involved in the transition of normal tissue into adenoma while neutrophil-mediated immunity was the most dysregulated in adenocarcinoma.

\section{Conclusion}


The present study preliminary uncovered the mechanism behind initiation and progression in duodenal carcinoma in FAP, and provided some scientific information for exploring novel therapeutic strategies for FAP patients with duodenal carcinoma.

\section{Background}

As a well-described autosomal dominant inherited syndrome, familial adenomatous polyposis (FAP) is caused by adenomatous polyposis coli (APC) gene mutation (Kim, Lee, Shim, \& Lee, 2014; Perea Garcia et al., 2019), which has a very high risk of developing gastrointestinal cancer (Half, Bercovich, \& Rozen, 2009). FAP is characterized by the formation of hundreds or thousands of adenomas in digestive tract (Hale et al., 2017), which often causes gastrointestinal bleeding, obstruction and other complications (Stevanato Filho et al., 2018). As known, if left treated, FAP will inevitably develop into colorectal cancer (CRC); thus, prophylactic colectomy remains a main strategy for the prevention of CRC in FAP patients (Lami et al., 2017; Vitellaro et al., 2020). In the past decades, increasing patients underwent prophylactic colectomy in early times, which substantially decreased the incidence of CRC (Koskenvuo, Ryynänen, \& Lepistö, 2020; Schneider, Schneider, Dalchow, Jakobeit, \& Möslein, 2015). Therefore, nowadays, the most frequent causes of death in FAP patients is mainly attributed to duodenal carcinoma (Schneider et al., 2015).

Despite most FAP patients exhibit limited duodenal polyposis, the relative risk of developing duodenal carcinoma of them is over 300 folds higher than that of the general population (Augustin et al., 2020). There are several managements of duodenal polyposis in preventing malignant transformation, which included surgical resection (Augustin, Moslim, Tang, \& Walsh, 2018) and chemoprevention (Delker et al., 2018). According to the degrees of organ preservation, there are commonly three operative techniques in duodenal polyposis therapy, including pancreatoduodenectomy (PD), pancreas-sparing duodenectomy (PSD) and Segmental duodenal resection (SDR) (Augustin et al., 2018). Several studies confirmed that these three operations could obviously increase cancer-free survival time of FAP patients with duodenal polyposis (Augustin et al., 2020; Augustin et al., 2018). However, such major surgeries are generally accompanied by a significant risk of morbidity and mortality, rendering decision-making challenging in clinic (van Heumen et al., 2012). Besides, surgical intervention may not completely eliminate duodenal polyposis since patients remain at risk for development of new adenoma in the proximal jejunum (Alderlieste, Bastiaansen, Mathus-Vliegen, Gouma, \& Dekker, 2013). In addition, chemoprevention with ursodeoxycholic acid (ursodiol) (Parc et al., 2012) or cyclooxygenase-2 (COX-2) inhibitors (Phillips et al., 2002) has exhibited disappointing results, leading to a reliance on endoscopic surveillance. It is required to explore a novel way to improve the prevention of malignant transformation in FAP patients with duodenal polyposis.

Multiple recent studies reported that a novel therapy, namely gene therapy, could specifically deliver genetic materials into cells of patients to prevent or treat various diseases, such as hemophilia (George, 2017) and cancers (Ginn, Amaya, Alexander, Edelstein, \& Abedi, 2018). Yet, the genetic regulatory mechanism of how benign polys develop into tumors in FAP patients with duodenal carcinoma has been 
seldom studied. Thus, a comprehensive analysis of understanding the molecular mechanism of duodenal carcinoma development in FAP patients is of great value.

Benefit from the rapid development of microarray and high-throughput sequencing technology, increasing potential biomarkers that contributed to disease progression have been identified (Ni et al., 2018; Zhou, Tang, Liu, An, \& Zhang, 2017). Several large and free public transcriptome databases, including GEO, TCGA, and CGGA databases, provide valuable gene expression data for the identification of potential biomarkers that related to diagnosis, prognosis or phenotype, and the construction of genome-wide coexpression networks, and the study of molecular mechanisms of pathogenesis (Hu et al., 2019; Ji et al., 2020). The weighted gene co-expression network analysis (WGCNA) is widely used in disease and other traits and gene association analysis, as it has the capacity of clustering genes with similar expression patterns and analyzing the relationship between modules and specific traits or phenotypes (Langfelder \& Horvath, 2008). In a newly study of cervical intraepithelial neoplasia (CIN), WGCNA was performed to identify six significant correlation modules and screen 31 candidate hub genes for CIN treatment (Zhang et al., 2020). Bioinformatics analysis could not only greatly improve the efficiency of research on biological functions, but also provide reliable information for exploring molecular mechanisms (J. Li et al., 2020; Pan et al., 2019).

At present, there are few studies exploring the molecular mechanism by which FAP trigger and regulate duodenal carcinoma. Hence, the present study sought to use the bioinformatics approaches to provide novel insights into the molecular mechanism of FAP developing into duodenal carcinoma. In this study, based on the gene expression profile from the GSE111156 dataset, differential expression analysis and WGCNA were performed to identify differentially expressed genes (DEGs) and key gene modules, respectively. The functional enrichment analysis was conducted to understand biological functions of DEGs and key module genes. Afterward, biological functions and pathways related to the immune system were further screened for providing the scientific information of immunotherapy in the context of duodenal carcinoma following FAP. Our findings may contribute to the prevention and therapy of duodenal carcinoma for FAP patients.

\section{Materials And Methods}

\section{Data collection}

The gene expression dataset GSE111156 downloaded from GEO database (https://www.ncbi.nlm.nih.gov/geo/query/acc.cgi?acc=GSE111156) was provided by Thiruvengadam et al. (2019). The dataset was composed of 48 samples, 36 from 12 FAP patients diagnosed with duodenal carcinoma (cases) and 12 from 12 FAP patients without duodenal carcinoma (controls). The study design was depicted in Fig. 1.

\section{Differential expression analysis}


The differential expression analysis was performed to identify DEGs from four comparisons by using an R package "limma" (ME et al., 2015). In this study, the threshold of defining DEGs was absolute log2 fold change $(F C)>0.263$ and $p$ value $<0.05$. The four comparisons were described in Fig. 1 .

\section{Construction of weighted gene co-expressed network}

By using the R package "WGCNA" (Langfelder \& Horvath, 2008), two co-expression networks were constructed respectively based on the expression data from case (contains three types of tissues: normal, adenoma and adenocarcinoma) or adenoma (from two types of FAP patients: case and control). In brief, the detection of outlier sample was carried out before constructing the adjacency matrix with Pearson's correlation coefficient matrix of all gene pairs. Afterward, when the degree of independence was 0.8 , soft threshold power $\beta$ was determined to construct a scale-free co-expression network with the adjacency matrix. Then the adjacency matrix was converted into a Topological Overlap Matrix (TOM). By using a TOM-based dissimilarity measure, the gene tree dendrogram was averagely linked hierarchically clustered, and genes with similar expression patterns were classified into the same modules. The modules with the high similarity of feature genes in the gene cluster dendrogram were merged according to an abline of 0.25 .

\section{Identification of the key modules}

By evaluating the potential correlation of genes with different traits, module eigengenes (MEs) could be utilized to determine the significance of each module. Besides, the correlation between genes and different clinical traits was defined as gene significance (GS), and average GS of all genes in a module was used to represent module significance (MS). For the studied trait, the module with the highest value of MS among all modules, which means the most significant correlation with this trait, was defined as the key module. The relationships of modules and traits were shown as a heatmap, and the strength of correlations was represented by the shades of color.

After identifying the key modules, a co-expression network within the key module was constructed by genes displaying module membership (MM) $\geq$ median and $G S \geq$ median, and visualized by using the network analyzer Cytoscape v3.7.2 (P et al., 2003). A plug-in of the Cytoscape, MCODE, was used to identify the hub genes that contained highly interconnected nodes in gene-gene interactional network.

\section{Functional enrichment analysis}

In this study, by using R package "clusterProfiler" (Yu, Wang, Han, \& He, 2012), functional enrichment analysis containing Gene Ontology (GO) enrichment analysis and Kyoto Encyclopedia of Genes and Genomes (KEGG) pathway analysis were carried out to understand the potential functions of the identified genes, thereby exploring the potential mechanism of disease development. The $p$ value $<0.5$ was set as the cut-off value, the q value as well as adjusted $p$ values were also generated.

\section{Results}




\section{Identification of DEGs in adenoma from case and control}

In total, 126 DEGs were identified according to the criteria of absolute log2 $\mathrm{FC}>0.263$ and $\mathrm{p}$ value $<0.05$, which included 13 up-regulated and 113 down-regulated genes (Table 1). The identified DEGs and corresponding FC as well as $\mathrm{p}$ value were presented in supplementary Table S1. The volcano plot containing all genes and heatmap depicting the top 30 DEGs were shown in Fig. 2A and B, respectively. After performing functional annotation of these DEGs, the results were recorded in supplementary Table S2, and the top 8 significant terms of GO and KEGG enrichment were visualized by dot plots (Fig. $2 \mathrm{C}$ and D). As we can see, the DEGs were significantly enriched in terpenoid metabolic process, isoprenoid metabolic process, and alcohol metabolic process. Besides, these DEGs were mainly involved in the pathways of chemical carcinogenesis, drug metabolism-cytochrome P450, and retinol metabolism.

Table 1

Results of differential expression analysis

\begin{tabular}{|lllll|}
\hline Comparison & \multicolumn{2}{l}{$\begin{array}{l}\text { Adenoma tissues from two } \\
\text { groups of FAP patients }\end{array}$} & \multicolumn{3}{l|}{$\begin{array}{l}\text { Three types of tissues from } \\
\text { FAP patients with duodenal carcinoma }\end{array}$} \\
\cline { 2 - 5 } & Case & Adenoma & Adenocarcinoma & $\begin{array}{l}\text { Adenocarcinoma } \\
\text { vs. }\end{array}$ \\
& vs. & vs. & vs. & normal \\
& control & normal & adenoma & 171 \\
\hline $\begin{array}{l}\text { Up- } \\
\text { regulated }\end{array}$ & 13 & 12 & 32 & 238 \\
$\begin{array}{l}\text { Down- } \\
\text { regulated }\end{array}$ & 113 & 17 & 18 & 409 \\
\hline Total & 126 & 29 & 50 & \\
\hline
\end{tabular}

\section{Screening of key modules associated with case or control}

As shown in Fig. 3A, no outlier sample was detected after sample clustering. To ensure a scale-free network, the soft threshold power $\beta$ was set as 18 (Fig. 3B). Through dynamic tree shearing method, the initial module was divided, followed by merged the modules based on abline equaled 0.25 (Fig. $3 \mathrm{C}$ ). A total of 16 modules were subsequently obtained. Except for the grey module, each module contains a group of feature genes with high similarity. Then, the correlation between clinical traits and each module was analyzed, and a heatmap was generated to visualize these relationships (Fig. 3D). Based on the heatmap, the blue module exhibited the most positive correlation with case (Pearson Cor $=0.62, p=$ 0.001), whereas the darkgreen module showed the most positive correlation with control (Pearson Cor $=$ $0.53, p=0.007)$. This means that the genes in these module are most relevant to the initiation of duodenal carcinoma in FAP patients. Hence, we selected these two modules as the key modules for further analysis. 


\section{Analysis of genes in the blue module associated with case}

The MM vs. GS in the blue module with cor $=0.74$ and $p<1 e-200$ was shown in Fig. 4A. A network containing 101 blue module genes and their multiple interactions were visualized by Cytoscape (Fig. 4B). Through MCODE, a plug-in of Cytoscape, a total of 11 hub genes were identified, which included RUSC2, CDH15, ABCA7, ZSWIM4, ADAM8, TERT, RADIL, UNC5A, RAB3IL1, AGAP3 and TP73. The G0 and KEGG pathway enrichment analysis were performed to better understand the biological significance of blue module genes, and the detailed information was presented in supplementary Table S3. As shown in Fig. 4C, genes of the blue module were mainly enriched in regulation of neuron projection development, modulation of chemical synaptic transmission, and regulation of trans-synaptic signaling. For KEGG pathway enrichment, these genes were significantly associated with neuroactive ligand-receptor interaction, calcium signaling pathway, and MAPK signaling pathway (Fig. 4D).

\section{Analysis of genes in the darkgreen module associated with control}

As shown in Fig. 5A, the correlation between GS for control and MM in darkgreen module reached 0.71 ( $p$ $=4.5 \mathrm{e}-46$ ), suggesting that the specific expression of genes in the darkgreen module are closely related to FAP without duodenal carcinoma. The gene-gene interaction network within the darkgreen module was shown in Fig. 5B. By using MCODE, we identified a total of 24 hub genes (MEP1B, SLC15A1, MGAM, CYP3A4, MS4A10, MEP1A, APOB, ABCG2, ALPI, MME, ASAH2, MME_AS1, TMPRSS15, ASAH2C, ADA, MY01A, SLC6A19, EPB41L3, PCSK5, CDHR5, DHRS11, ASAH2B, SLC46A1, and CREB3L3). Afterward, the functional annotation of genes in the darkgreen module revealed that these genes were mainly involved in fatty acid metabolic process, lipid catabolic process, protein digestion and absorption, fat digestion and absorption, and chemical carcinogenesis (Fig. 5C and D).

\section{Identification of DEGs between different tissues from case}

The results of differential expression analysis were depicted in Table 1, the detailed information of which was summarized in supplementary Table S1.

In the comparison of adenoma and normal, only 29 DEGs were identified, which included 12 up-regulated and 17 down-regulated genes (Fig. 6A). The heatmap depicting the top 30 DEGs was shown in Figure S1A. In order to understand the biological pathways involved, we conducted the functional annotation for these DEGs. As shown in Fig. 6B, these DEGs were mainly associated with the metabolism of many terpenoids, such as retinoid metabolic process, diterpenoid metabolic process, terpenoid metabolic process, as well as isoprenoid metabolic process. The KEGG analysis showed that these DEGs were mostly involved in vitamin digestion and absorption, folate biosynthesis, and protein digestion and absorption (Fig. 6C). 
The volcano plot of the DEGs between adenocarcinoma and adenoma was shown in Fig. 6D. Among 50 DEDs, 32 were up-regulated while 18 were down-regulated. The heatmap of the top 30 DEGs was depicted in Figure S1B. As shown in Fig. $6 \mathrm{E}$ and F, these DEGs were mainly enriched in extracellular matrix organization, extracellular structure organization, protein digestion and absorption, ECM-receptor interaction, and AGE-RAGE signaling pathway in diabetic complications.

After comparing the gene expression profile between adenocarcinoma and normal from case, we identified a total of 409 DEGs including 171 up-regulated and 238 down-regulated genes (Fig. 6G). The top $30 \mathrm{DEGs}$ were displayed as a heatmap in Figure S1C. The functional enrichment analysis was also conducted. As shown in Fig. 6H, these DEGs in GO analysis were related to extracellular matrix organization, extracellular structure organization, hormone metabolic process, and terpenoid metabolic process. According to KEGG analysis, we found that these DEGs were significantly enriched in chemical carcinogenesis, drug metabolism - cytochrome P450, and metabolism of xenobiotics by cytochrome P450 (Fig. 6l).

\section{Identification of the key module associated with normal, adenoma, and adenocarcinoma in case}

Sample dendrogram and trait heatmap was shown in Fig. 7A, suggesting no outlier within 36 samples. Figure 7B showed that the lowest power of the scale-free topology fit index on 0.8 was 20 . Thus soft threshold power $\beta$ was set as 20 for subsequent analysis. As shown in Fig. 7C and D, after merging the original modules based on abline $=0.25$, a total of 15 modules were finally got. According to the heatmap of module-trait relationships (Fig. 7E), the "normal" trait was most positively related to the orange module (Pearson Cor $=0.73, p=3 e-0.7$ ), and the "adenocarcinoma" trait was most positively related to the drakorange module (Pearson Cor $=0.64, p=3 e-0.5$ ). Thus, the orange and drakorange modules were considered as the key module of normal and adenocarcinoma, respectively. Besides, we found that no modules exhibited a significantly positive correlation with the "adenoma" trait. Therefore, the cyan module, which was most negatively associated with adenoma, was selected as the key module of adenoma.

\section{Analysis of genes in the orange module associated with normal}

The MM vs. GS in the blue module with cor $=0.74$ and $p=1.7 \mathrm{e}-117$ was shown in Fig. 8A. Through MCODE, we identified a total of 26 hub genes within the orange module, including IGHV3_30, IGKV3D_11, IGKV1D_37, IGHV3_15, IGHV3_21, IGHJ5, IGHV1_18, IGKV3D_20, IGKV1D_33, IGKV3D_7, IGKV3_11, IGKV2D_29, IGKV1_9, IGHV1_69, IGKV3D_15, IGKV1_17, IGKV1D_16, IGKV1_27, IGKC, IGKV1D_39, IGKV1_6, IGKV1_8, IGKV1_5, IGKV1D_42, IGKV1D_27, and IGHV3_48 (Fig. 8B). The GO and KEGG pathway enrichment analysis were performed to better understand the biological significance of orange module genes, and the detailed information was listed in supplementary Table S6. As shown in Fig. 8C, genes in the orange module were mainly enriched in GO terms of small molecule catabolic process, fatty acid 
metabolic process, and lipid catabolic process. The major KEGG pathways were chemical carcinogenesis, drug metabolism - cytochrome P450, and metabolism of xenobiotics by cytochrome P450 (Fig. 8D).

\section{Analysis of genes in the cyan module associated with adenoma}

As shown in Fig. 9A, the correlation between GS for control and MM in cyan module was $0.24(p=2.5 e-$ 08). According to the network that generated by Cytoscape with MCODE, we screened 21 hub genes of cyan module, which included COL1A2, PDGFRB, GUCY1A3, COL3A1, PXDN, RAB31, BGN, COL4A2, COL5A2, MMP2, COL1A1, VCAN, ITGA11, AEBP1, FBN1, ANTXR1, ASPN, COL6A3, SPARC, LAMB2, and CDH11 (Fig. 9B). Functional analysis of the cyan module genes revealed that these genes were mainly involved in extracellular matrix organization, extracellular structure organization, and ossification (Fig. 9C). The most representative KEGG pathways are focal adhesion, ECM-receptor interaction, and PI3K-Akt signaling pathway (Fig. 9D). More detailed information of functional annotation for this module is represented in supplementary Table S6.

\section{Analysis of genes in the darkorange module associated with adenocarcinoma}

A scatter plot (cor $=0.64$ and $p=1.6 e-76$ ) of GS for adenocarcinoma vs MM in the darkorange module was depicted in Fig. 10A. Afterward, a total of 19 hub genes (TSTA3, AKT1, LSM4, ADRBK1, MIR663A, PRMT1, BSG, SMARCD2, UBA1, DDX39A, RNF126, U2AF2, PSMD3, JUP, PPIF, PPP4C, SLC12A7, RAD23A, and TMEM259) were identified in the darkorange module (Fig. 10B). Based on the results of GO analysis, genes in the darkorange module were mainly enriched in regulation of cell morphogenesis, neutrophil mediated immunity, and extracellular matrix organization (Fig. 10C). Furthermore, these genes were suggested related to KEGG pathways of focal adhesion, regulation of actin cytoskeleton, and phagosome (Fig. 10D).

\section{Functional roles of immune genes in different traits}

In order to identify the terms directly linked to immune response, we searched the results of GO and KEGG analyses for the corresponding terms. The detailed information was given in supplementary Table S4 and S7.

The DEGs identified between case and control might play a potential role in the initiation of FAP developing into duodenal carcinoma. We found that seven terms were related to immune response in their functional results, which were mainly associated with the T cell proliferation. Besides, only four immune related terms were found in the functional annotation of blue module genes associated with case. For the darkgreen module genes associated with control, the total of immune related terms in their functional annotation was seven, and these terms were mainly related to neutrophil. These data suggested that whether FAP developed into duodenal carcinoma was less involved in immune response. 
Performing the analyses between three different tissues from FAP patients with duodenal carcinoma might provide novel insights into the mechanism of duodenal carcinoma. Hence, we also investigated the immunological function of genes associated with different comparisons or traits in order to explore the possibility of immunotherapy for treating FAP with duodenal carcinoma. Notably, the DEGs identified in three comparisons all mainly related with immunological process of leukocyte migration, suggesting that this process has crucial significance in the progression of FAP with duodenal carcinoma. Moreover, the genes associated with normal (orange module genes) and the genes associated with adenoma (cyan module genes) were also mainly involved in the biological process of leukocyte migration. The genes associated with adenocarcinoma (darkorange module genes) were mainly enriched in neutrophil mediated immunity. Furthermore, in KEGG pathway enrichment results, we found that cyan module genes and the darkorange module genes were both significantly involved in phagosome.

\section{Discussion}

According to previous studies, the occurrence of duodenal adenoma for FAP patients is about $90 \%$ (Bülow et al., 2004), and the risk of such patients developing duodenal carcinoma is estimated to be 312\% (Pittayanon, Imraporn, Rerknimitr, \& Kullavanijaya, 2014). However, the underlying mechanisms driving duodenal adenoma into carcinoma are not fully understood. Thiruvengadam et al. (2019) performed transcriptional profiling (GSE111156) to reveal the duodenal adenoma-carcinoma sequence and uncover changes differentiating patients with FAP with and without duodenal carcinoma. Among DEGs they identified, only the genes that have already been revealed in previous studies of FAP were chosen for further analyses, which might overlook some important genes or mechanisms. Based on the same dataset, this study performed bioinformatics analyses (differential expression analysis and WGCNA) with the gene expression profiles of FAP patients without duodenal carcinoma and FAP patients with duodenal carcinoma in order to reveal the mechanism behind duodenal carcinoma initiation in FAP patients. Meanwhile, the same analyses were conducted to explore genetic markers and pathways related to the duodenal tissue of normal, adenoma and adenocarcinoma from FAP patients with duodenal carcinoma, which might provide certain scientific information to the gene therapy for FAP with duodenal carcinoma.

Our study revealed that the DEGs identified from the comparison of case and control were most related to chemical carcinogenesis pathway and multiple metabolic processes, hinting that the abnormal metabolism of multiple active substances triggered chemical carcinogenesis pathway, thereby initiating the development of FAP into duodenal carcinoma. As reported, alcohol metabolism is a major contributor to the increased risk of certain cancers, such as colorectal carcinoma (Rossi, Jahanzaib Anwar, Usman, Keshavarzian, \& Bishehsari, 2018) and hepatocellular carcinoma (Zahid, Yao, Khan, Raza, \& Gou, 2019). Alcohol metabolism products have been related to an increased level of NADH, which results in histone modifications and changes in gene expression that contribute to influence cancer susceptibility ( $\mathrm{Na}$ \& Lee, 2017). There is not much data published on the role of terpenoids metabolism in carcinogenesis. Actually, terpenoids are crucial for our bodies to produce many essential compounds, such as vitamin A and retinol (Pichersky \& Raguso, 2018); and the changed level of retinol in serum has been proved to be 
correlated with the progression of several cancers (Maalmi et al., 2018; Rosa et al., 2019). Unlike the differential expression analysis, WGCNA could screen highly synergistically altered gene sets and further identify those most relevant to traits of interest. The results of WGCNA provided us with other information. The genes in the blue module related to FAP case exhibited strong correlation with regulation of neuron projection development and neuroactive ligand-receptor interaction. Several previous studies demonstrated that the enteric nervous system plays a critical role in the regulation of intestinal homeostasis (Le Berre-Scoul et al., 2017; Neunlist et al., 2014). A recent in vivo study conducted by Yuan et al. (2020) demonstrated that enteric glial plays a critical role in modulating the origin of colorectal carcinoma. Hence, we speculated that the duodenal carcinoma in FAP patients was probably initiated by the abnormal metabolism of terpenoids and enteric nervous system. For the genes in the drakgreen module associated with FAP control, we found that they were enriched in processes and pathways that are closely related to lipid metabolism, such as fatty acid metabolic process, lipid catabolic process, and fat digestion and absorption pathway. Many studies have corroborated the association between malignancies and altered metabolism of lipids (Beloribi-Djefaflia, Vasseur, \& Guillaumond, 2016; Pakiet, Kobiela, Stepnowski, Sledzinski, \& Mika, 2019). As lipids are essential for the proliferation of cancer cells (Zaytseva et al., 2015), abnormal lipid metabolism in FAP patients may present a warning for carcinogenesis. Therefore, some biological processes involved in the synthesis and storage of lipid might be used as targets to prevent FAP developing into duodenal carcinoma.

The analyses of three tissues from FAP case samples indirectly revealed the potential mechanism of normal-adenoma-adenocarcinoma progression in FAP with duodenal carcinoma. In the differential expression analysis, only 29 and 50 DEGs were respectively identified in comparisons of normal and adenoma, adenoma and adenocarcinoma; but there are 409 DEGs in the comparison of normal and adenocarcinoma. That means, the expression of some genes was changed significantly after two transitions, and altered expression of such genes was not obvious during each transition. In other words, differential expression analysis might exclude some important genes whose expression level changes little, but play a crucial role in the transition. WGCNA can avoid this issue. Hence, we combined the results of the two analyses and found that the malignant transformation in FAP patient with duodenal carcinoma might be involved in the following processes. Initially, the abnormal metabolism of lipid and terpenoids break the homeostasis of duodenum, rendering to the development of adenoma. Afterward, abnormal extracellular matrix (ECM) dynamics play a critical role in the transition of adenoma into adenocarcinoma. ECM is known as a major structural component of the tumor microenvironment (Walker, Mojares, \& Del Río Hernández, 2018). ECM receptor interaction stimulates focal adhesion, in turn to the regulation of the PI3K-Akt signaling pathway (Aboubakar Nana et al., 2019). Notably, our results suggested that adenoma-adenocarcinoma sequence was also closely related to ossification. A previous study reported that tumor-derived ECM is biochemically distinct in its composition and is stiffer compared to normal ECM (Nallanthighal, Heiserman, \& Cheon, 2019). Finally, in the adenocarcinoma, the ECM dynamic is still aberrant, and the other biological processes and pathways that might contribute to cancer progression have been further triggered, which included regulation of cell morphogenesis, neutrophil mediated immunity, and HIF-1 signaling pathway. Hypoxia is a hallmark of the tumor 
microenvironment (TME) in most solid tumors, which could trigger HIF-1 signaling pathway, thereby promoting a more aggressive and metastatic phenotype (Huang, Lin, \& Taniguchi, 2017). Numerous studies revealed that HIF-1 signaling pathway is closely related to malignant progression of several cancers, including cancer metastasis, invasiveness, and resistance to treatment (Huang et al., 2017; Rankin \& Giaccia, 2016). Thus, targeting this pathway could be represented a promising strategy for inhibiting the progression of duodenal carcinoma and improving patients outcome. In the process of tumor development, in addition to hypoxia, multiple complex changes take place in the TME, including the immune response and infiltration of various immune cells (Bindea et al., 2013), especially neutrophils (Uribe-Querol \& Rosales, 2015). Emerging evidence demonstrated that neutrophils expand both in the tumor microenvironment and systemically, and trigger the proliferation and metastasis of tumor cells, leading to a poor prognosis of patients with solid tumor (Coffelt, Wellenstein, \& de Visser, 2016; CoolsLartigue et al., 2013; S. Li et al., 2019). We also found that neutrophils might play a facilitative role in the progression of duodenal carcinoma in FAP patients, suggesting the potential of neutrophils as clinical biomarkers and therapeutic targets.

To date, immunotherapy is recognized as a promising avenue in the treatment of cancer, but its efficacy depends on the understanding of mechanism behind immune response during cancer development. Making use of the results acquired in this study, we found that the transition of "normal-adenoma" was mainly related to the immune response of leukocyte migration. It is already known that leukocyte migration plays a key role in the systemic distribution of immune cells (David \& Kubes, 2019). Phagosome formation is a critical factor for cancer initiation (Wang et al., 2020), which corroborated with our finding that the phagosome pathway was activated during the transition of "adenomaadenocarcinoma" in FAP patients with duodenal carcinoma. Moreover, the process of neutrophil mediated immunity exhibited a significant correlation with adenocarcinoma in this study. These data prompted that the processes of leukocyte migration and phagosome could be used as a therapeutic target of immunotherapy for FAP patients with duodenal carcinoma; and neutrophil suppression may improve the therapeutic effect of immunotherapy.

Collectively, the present study exploited the patho-mechanism of FAP with duodenal carcinoma and provides a novel insight into immunotherapy in this disease. There are some limitations in this study. First, the sample size was relatively small. Before the end of this study, only one dataset that contained normal samples, adenoma samples and adenocarcinoma samples from FAP patient with duodenal carcinoma was found. Besides, our finding is a preliminary step to reveal underlying mechanism for the development of FAP with duodenal carcinoma; further experimental verification should be conducted to obtain a solid result.

\section{Conclusion}

The current study revealed that terpenoids and lipid metabolism might play a critical role in the initiation of duodenal carcinoma in FAP patients. Our study also found that ECM related processes were a main contributor for driving the progression of duodenal carcinoma in FAP. The results of this research help us 
better understand the development of duodenal carcinoma in FAP, and provide some scientific information for exploring novel therapeutic strategies for FAP patients with duodenal carcinoma.

\section{Declarations}

\section{Ethics approval and consent to participate}

Not applicable.

\section{Consent for publication}

Not applicable.

\section{Availability of data and materials}

All data generated or analyzed during this study are included in this published article.

\section{Competing interests}

The authors declare that they have no competing interests.

\section{Funding}

This work was supported by the National Natural Science Foundation of China (No. 81760511 and No. 81960100); Applied Basic Foundation of Yunnan Province (No. 202001AT070009); Yunnan Health Training Project of High Level Talents (No. D-2019032).

\section{Authors' contributions}

WQL wrote the manuscript and interpreted the data. WQL, XYM, XTL, LL, ZYK and JY contributed in data analysis and interpretation. WQL and JY conceptualized and designed the study. All the authors have read and approved the final version of the manuscript.

\section{Acknowledgements}

Not applicable.

\section{References}

Aboubakar Nana, F., Lecocq, M., Ladjemi, M. Z., Detry, B., Dupasquier, S., Feron, O., . . Ocak, S. (2019). Therapeutic Potential of Focal Adhesion Kinase Inhibition in Small Cell Lung Cancer. Mol Cancer Ther, 18(1), 17-27. doi:10.1158/1535-7163.Mct-18-0328

Alderlieste, Y. A., Bastiaansen, B. A., Mathus-Vliegen, E. M., Gouma, D. J., \& Dekker, E. (2013). High rate of recurrent adenomatosis during endoscopic surveillance after duodenectomy in patients with familial 
adenomatous polyposis. Fam Cancer, 12(4), 699-706. doi:10.1007/s10689-013-9648-5

Augustin, T., Moslim, M. A., Cengiz, T. B., El-Hayek, K., Simon, R., Bhatt, A., ... Matthew Walsh, R. (2020). Survival outcomes after surgical management of sporadic or familial adenomatous polyposis associated duodenal cancer. J Surg Oncol, 122(6), 1132-1144. doi:10.1002/jso.26131

Augustin, T., Moslim, M. A., Tang, A., \& Walsh, R. M. (2018). Tailored surgical treatment of duodenal polyposis in familial adenomatous polyposis syndrome. Surgery, 163(3), 594-599.

doi:10.1016/j.surg.2017.10.035

Beloribi-Djefaflia, S., Vasseur, S., \& Guillaumond, F. (2016). Lipid metabolic reprogramming in cancer cells. Oncogenesis, 5(1), e189. doi:10.1038/oncsis.2015.49

Bindea, G., Mlecnik, B., Tosolini, M., Kirilovsky, A., Waldner, M., Obenauf, A. C., . . Galon, J. (2013). Spatiotemporal dynamics of intratumoral immune cells reveal the immune landscape in human cancer. Immunity, 39(4), 782-795. doi:10.1016/j.immuni.2013.10.003

Bülow, S., Björk, J., Christensen, I. J., Fausa, O., Järvinen, H., Moesgaard, F., . . Group, D. A. F. S. (2004). Duodenal adenomatosis in familial adenomatous polyposis. Gut, 53(3), 381-386. Retrieved from https://pubmed.ncbi.nlm.nih.gov/14960520

https://www.ncbi.nlm.nih.gov/pmc/articles/PMC1773976/. doi:10.1136/gut.2003.027771

Coffelt, S. B., Wellenstein, M. D., \& de Visser, K. E. (2016). Neutrophils in cancer: neutral no more. Nat Rev Cancer, 16(7), 431-446. doi:10.1038/nrc.2016.52

Cools-Lartigue, J., Spicer, J., McDonald, B., Gowing, S., Chow, S., Giannias, B., . . Ferri, L. (2013). Neutrophil extracellular traps sequester circulating tumor cells and promote metastasis. J Clin Invest, 123(8), 3446-3458. doi:10.1172/jci67484

David, B. A., \& Kubes, P. (2019). Exploring the complex role of chemokines and chemoattractants in vivo on leukocyte dynamics. Immunol Rev, 289(1), 9-30. doi:10.1111/imr.12757

Delker, D. A., Wood, A. C., Snow, A. K., Samadder, N. J., Samowitz, W. S., Affolter, K. E., . . Neklason, D. W. (2018). Chemoprevention with Cyclooxygenase and Epidermal Growth Factor Receptor Inhibitors in Familial Adenomatous Polyposis Patients: mRNA Signatures of Duodenal Neoplasia. Cancer Prev Res (Phila), 17(1), 4-15. doi:10.1158/1940-6207.Capr-17-0130

George, L. A. (2017). Hemophilia gene therapy comes of age. Blood Adv, 1(26), 2591-2599. doi:10.1182/bloodadvances.2017009878

Ginn, S. L., Amaya, A. K., Alexander, I. E., Edelstein, M., \& Abedi, M. R. (2018). Gene therapy clinical trials worldwide to 2017: An update. J Gene Med, 20(5), e3015. doi:10.1002/jgm.3015 
Hale, V. L., Chen, J., Johnson, S., Harrington, S. C., Yab, T. C., Smyrk, T. C., . . Chia, N. (2017). Shifts in the Fecal Microbiota Associated with Adenomatous Polyps. Cancer Epidemiol Biomarkers Prev, 26(1), 85-94. Retrieved from https://www.ncbi.nlm.nih.gov/pubmed/27672054. doi:10.1158/1055-9965.EPI-16-0337

Half, E., Bercovich, D., \& Rozen, P. (2009). Familial adenomatous polyposis. Orphanet J Rare Dis, 4, 22. doi:10.1186/1750-1172-4-22

Hu, J., Zhou, L., Song, Z., Xiong, M., Zhang, Y., Yang, Y., . . Chen, Z. (2019). The identification of new biomarkers for bladder cancer: A study based on TCGA and GEO datasets. J Cell Physiol. doi:10.1002/jcp.28208

Huang, Y., Lin, D., \& Taniguchi, C. M. (2017). Hypoxia inducible factor (HIF) in the tumor microenvironment: friend or foe? Sci China Life Sci, 60(10), 1114-1124. doi:10.1007/s11427-017-9178-y Ji, B., Chen, L., Cai, Q., Guo, Q., Chen, Z., \& He, D. (2020). Identification of an 8-miRNA signature as a potential prognostic biomarker for glioma. PeerJ, 8, e9943. doi:10.7717/peerj.9943

Kim, H. B., Lee, Y. J., Shim, J. Y., \& Lee, H. R. (2014). The association between coronary calcification and adenomatous polyps of colon in Korean adults. Clin Res Hepatol Gastroenterol, 38(5), 649-654. Retrieved from https://www.ncbi.nlm.nih.gov/pubmed/24969685. doi:10.1016/j.clinre.2014.01.004

Koskenvuo, L., Ryynänen, H., \& Lepistö, A. (2020). Timing of prophylactic colectomy in familial adenomatous polyposis. Colorectal Dis, 22(11), 1553-1559. doi:10.1111/codi.15151

Lami, G., Galli, A., Macrì, G., Dabizzi, E., Biagini, M. R., Tarocchi, M., .. Polvani, S. (2017). Gastric and duodenal polyps in familial adenomatous polyposis patients: Conventional endoscopy vs virtual chromoendoscopy (fujinon intelligent color enhancement) in dysplasia evaluation. World journal of clinical oncology, 8(2), 168-177. Retrieved from https://pubmed.ncbi.nlm.nih.gov/28439498

https://www.ncbi.nlm.nih.gov/pmc/articles/PMC5385438/. doi:10.5306/wjco.v8.i2.168

Langfelder, P., \& Horvath, S. (2008). WGCNA: an R package for weighted correlation network analysis. BMC bioinformatics, 9, 559. doi:10.1186/1471-2105-9-559

Le Berre-Scoul, C., Chevalier, J., Oleynikova, E., Cossais, F., Talon, S., Neunlist, M., \& Boudin, H. (2017). A novel enteric neuron-glia coculture system reveals the role of glia in neuronal development. $J$ Physiol, 595(2), 583-598. doi:10.1113/jp271989

Li, J., Lu, L., Zhang, Y. H., Xu, Y., Liu, M., Feng, K., . . Cai, Y. D. (2020). Identification of leukemia stem cell expression signatures through Monte Carlo feature selection strategy and support vector machine. Cancer Gene Ther, 27(1-2), 56-69. doi:10.1038/s41417-019-0105-y

Li, S., Cong, X., Gao, H., Lan, X., Li, Z., Wang, W., . . Xue, Y. (2019). Tumor-associated neutrophils induce EMT by IL-17a to promote migration and invasion in gastric cancer cells. J Exp Clin Cancer Res, 38(1), 6. 
Maalmi, H., Walter, V., Jansen, L., Owen, R. W., Ulrich, A., Schöttker, B., . . Brenner, H. (2018). DoseResponse Relationship between Serum Retinol Levels and Survival in Patients with Colorectal Cancer: Results from the DACHS Study. Nutrients, 10(4). doi:10.3390/nu10040510

ME, R., B, P., D, W., Y, H., CW, L., W, S., \& GK, S. (2015). limma powers differential expression analyses for RNA-sequencing and microarray studies. Nucleic acids research, 43(7), e47. doi:10.1093/nar/gkv007

Na, H. K., \& Lee, J. Y. (2017). Molecular Basis of Alcohol-Related Gastric and Colon Cancer. Int J Mol Sci, 18(6). doi:10.3390/ijms18061116

Nallanthighal, S., Heiserman, J. P., \& Cheon, D. J. (2019). The Role of the Extracellular Matrix in Cancer Stemness. Front Cell Dev Biol, 7, 86. doi:10.3389/fcell.2019.00086

Neunlist, M., Rolli-Derkinderen, M., Latorre, R., Van Landeghem, L., Coron, E., Derkinderen, P., \& De Giorgio, R. (2014). Enteric glial cells: recent developments and future directions. Gastroenterology, 147(6), 12301237. doi:10.1053/j.gastro.2014.09.040

Ni, M., Liu, X., Wu, J., Zhang, D., Tian, J., Wang, T., . . Zhang, X. (2018). Identification of Candidate Biomarkers Correlated With the Pathogenesis and Prognosis of Non-small Cell Lung Cancer via Integrated Bioinformatics Analysis. Front Genet, 9, 469. doi:10.3389/fgene.2018.00469

P, S., A, M., O, O., NS, B., JT, W., D, R., . . T, I. (2003). Cytoscape: a software environment for integrated models of biomolecular interaction networks. Genome research, 13(11), 2498-2504.

doi:10.1101/gr.1239303

Pakiet, A., Kobiela, J., Stepnowski, P., Sledzinski, T., \& Mika, A. (2019). Changes in lipids composition and metabolism in colorectal cancer: a review. Lipids in health and disease, 18(1), 29-29. Retrieved from https://pubmed.ncbi.nlm.nih.gov/30684960

https://www.ncbi.nlm.nih.gov/pmc/articles/PMC6347819/. doi:10.1186/s12944-019-0977-8

Pan, X., Zeng, T., Yuan, F., Zhang, Y. H., Chen, L., Zhu, L., . . Cai, Y. D. (2019). Screening of Methylation Signature and Gene Functions Associated With the Subtypes of Isocitrate Dehydrogenase-Mutation Gliomas. Front Bioeng Biotechnol, 7, 339. doi:10.3389/fbioe.2019.00339

Parc, Y., Desaint, B., Fléjou, J. F., Lefèvre, J. H., Serfaty, L., Vienne, A., . . Tiret, E. (2012). The effect of ursodesoxycholic acid on duodenal adenomas in familial adenomatous polyposis: a prospective randomized placebo-control trial. Colorectal Dis, 14(7), 854-860. doi:10.1111/j.1463-1318.2011.02816.x

Perea Garcia, J., Arribas, J., Canete, A., Garcia, J. L., Alvaro, E., Tapial, S., . . Gonzalez-Sarmiento, R. (2019). Association of Polyps with Early-Onset Colorectal Cancer and Throughout Surveillance: Novel 
Clinical and Molecular Implications. Cancers (Basel), 11(12). Retrieved from

https://www.ncbi.nlm.nih.gov/pubmed/31795313. doi:10.3390/cancers11121900

Phillips, R. K., Wallace, M. H., Lynch, P. M., Hawk, E., Gordon, G. B., Saunders, B. P., . . Steinbach, G. (2002). A randomised, double blind, placebo controlled study of celecoxib, a selective cyclooxygenase 2 inhibitor, on duodenal polyposis in familial adenomatous polyposis. Gut, 50(6), 857-860. doi:10.1136/gut.50.6.857

Pichersky, E., \& Raguso, R. A. (2018). Why do plants produce so many terpenoid compounds? New Phytol, 220(3), 692-702. doi:10.1111/nph.14178

Pittayanon, R., Imraporn, B., Rerknimitr, R., \& Kullavanijaya, P. (2014). Advances in diagnostic endoscopy for duodenal, including ampullary, adenoma. Dig Endosc, 26 Supp/ 2, 10-15. doi:10.1111/den.12244

Rankin, E. B., \& Giaccia, A. J. (2016). Hypoxic control of metastasis. Science, 352(6282), 175-180. doi:10.1126/science.aaf4405

Rosa, C., Franca, C., Lanes Vieira, S., Carvalho, A., Penna, A., Nogueira, C., . . Ramalho, A. (2019). Reduction of Serum Concentrations and Synergy between Retinol, $\beta$-Carotene, and Zinc According to Cancer Staging and Different Treatment Modalities Prior to Radiation Therapy in Women with Breast Cancer. Nutrients, 11(12). doi:10.3390/nu11122953

Rossi, M., Jahanzaib Anwar, M., Usman, A., Keshavarzian, A., \& Bishehsari, F. (2018). Colorectal Cancer and Alcohol Consumption-Populations to Molecules. Cancers (Basel), 10(2). doi:10.3390/cancers 10020038

Schneider, R., Schneider, C., Dalchow, A., Jakobeit, C., \& Möslein, G. (2015). Prophylactic surgery in familial adenomatous polyposis (FAP)-a single surgeon's short- and long-term experience with hand-assisted proctocolectomy and smaller J-pouches. Int J Colorectal Dis, 30(8), 1109-1115. doi:10.1007/s00384-0152223-9

Stevanato Filho, P. R., Aguiar Junior, S., Begnami, M. D., Ferreira, F. O., Nakagawa, W. T., Spencer, R., . . Lopes, A. (2018). Estrogen Receptor beta as a Prognostic Marker of Tumor Progression in Colorectal Cancer with Familial Adenomatous Polyposis and Sporadic Polyps. Pathol Oncol Res, 24(3), 533-540. Retrieved from https://www.ncbi.nlm.nih.gov/pubmed/28681123. doi:10.1007/s12253-017-0268-5

Thiruvengadam, S. S., O’Malley, M., LaGuardia, L., Lopez, R., Wang, Z., Shadrach, B. L., . . Burke, C. A. (2019). Gene Expression Changes Accompanying the Duodenal Adenoma-Carcinoma Sequence in Familial Adenomatous Polyposis. Clin Trans/ Gastroenterol, 10(6), e00053. doi: $10.14309 /$ ctg. 0000000000000053

Uribe-Querol, E., \& Rosales, C. (2015). Neutrophils in Cancer: Two Sides of the Same Coin. J Immunol Res, 2015, 983698. doi:10.1155/2015/983698 
van Heumen, B. W., Nieuwenhuis, M. H., van Goor, H., Mathus-Vliegen, L. E., Dekker, E., Gouma, D. J., . . . Nagengast, F. M. (2012). Surgical management for advanced duodenal adenomatosis and duodenal cancer in Dutch patients with familial adenomatous polyposis: a nationwide retrospective cohort study. Surgery, 151(5), 681-690. doi:10.1016/j.surg.2011.12.008

Vitellaro, M., Piozzi, G., Signoroni, S., Ricci, M. T., Ciniselli, C. M., Cardani, A., . . Biasoni, D. (2020). Shortterm and long-term outcomes after preventive surgery in adolescent patients with familial adenomatous polyposis. Pediatr Blood Cancer, 67(3), e28110. doi:10.1002/pbc.28110

Walker, C., Mojares, E., \& Del Río Hernández, A. (2018). Role of Extracellular Matrix in Development and Cancer Progression. Int J Mol Sci, 19(10). doi:10.3390/ijms19103028

Wang, X., Liu, G., Sheng, N., Zhang, M., Pan, X., Liu, S., . . Xu, J. (2020). Peptidome characterization of ovarian cancer serum and the identification of tumor suppressive peptide ZYX(36-58). Ann Trans/ Med, 8(15), 925. doi:10.21037/atm-20-2018

Yu, G., Wang, L.-G., Han, Y., \& He, Q.-Y. (2012). clusterProfiler: an R package for comparing biological themes among gene clusters. OMICS, 16(5), 284-287. Retrieved from

https://pubmed.ncbi.nlm.nih.gov/22455463

https://www.ncbi.nlm.nih.gov/pmc/articles/PMC3339379/. doi:10.1089/omi.2011.0118

Yuan, R., Bhattacharya, N., Kenkel, J. A., Shen, J., DiMaio, M. A., Bagchi, S., . . Engleman, E. G. (2020). Enteric Glia Play a Critical Role in Promoting the Development of Colorectal Cancer. Frontiers in oncology, 10, 595892-595892. Retrieved from https://pubmed.ncbi.nlm.nih.gov/33282743

https://www.ncbi.nlm.nih.gov/pmc/articles/PMC7691584/. doi:10.3389/fonc.2020.595892

Zahid, K. R., Yao, S., Khan, A. R. R., Raza, U., \& Gou, D. (2019). mTOR/HDAC1 Crosstalk Mediated Suppression of ADH1A and ALDH2 Links Alcohol Metabolism to Hepatocellular Carcinoma Onset and Progression in silico. Front Oncol, 9, 1000. doi:10.3389/fonc.2019.01000

Zaytseva, Y. Y., Harris, J. W., Mitov, M. I., Kim, J. T., Butterfield, D. A., Lee, E. Y., . . Evers, B. M. (2015). Increased expression of fatty acid synthase provides a survival advantage to colorectal cancer cells via upregulation of cellular respiration. Oncotarget, 6(22), 18891-18904. doi:10.18632/oncotarget.3783

Zhang, X., Bai, J., Yuan, C., Long, L., Zheng, Z., Wang, Q., . . Zhou, Y. (2020). Bioinformatics analysis and identification of potential genes related to pathogenesis of cervical intraepithelial neoplasia. Journal of Cancer, 11(8), 2150-2157. Retrieved from https://pubmed.ncbi.nlm.nih.gov/32127942

https://www.ncbi.nlm.nih.gov/pmc/articles/PMC7052918/. doi:10.7150/jca.38211

Zhou, D., Tang, W., Liu, X., An, H.-X., \& Zhang, Y. (2017). Clinical verification of plasma messenger RNA as novel noninvasive biomarker identified through bioinformatics analysis for lung cancer. Oncotarget, 


\section{Figures}

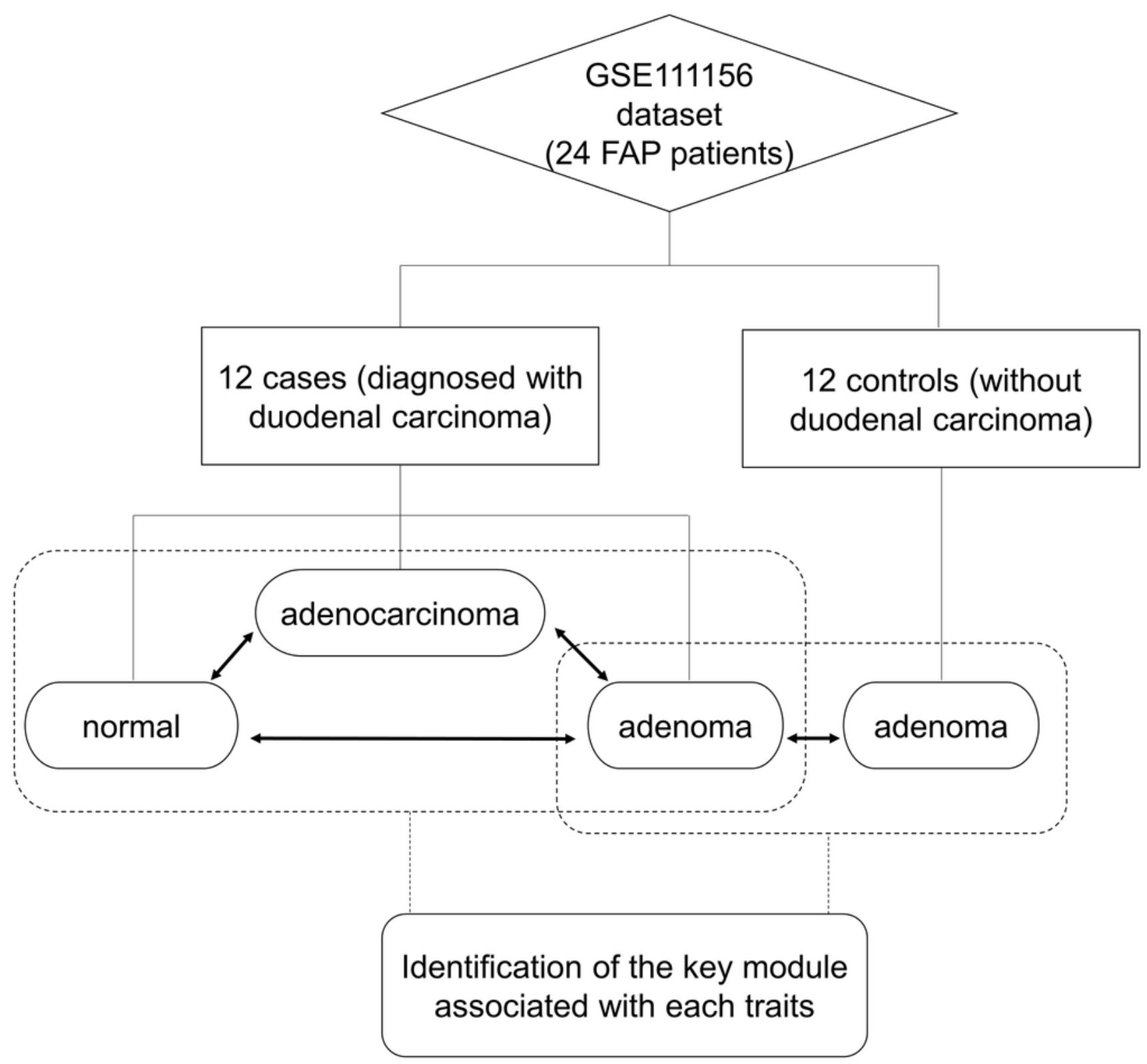

$\longleftrightarrow$ Represents differential expression analysis

\section{Represents WGCNA}


The design of this study.

A
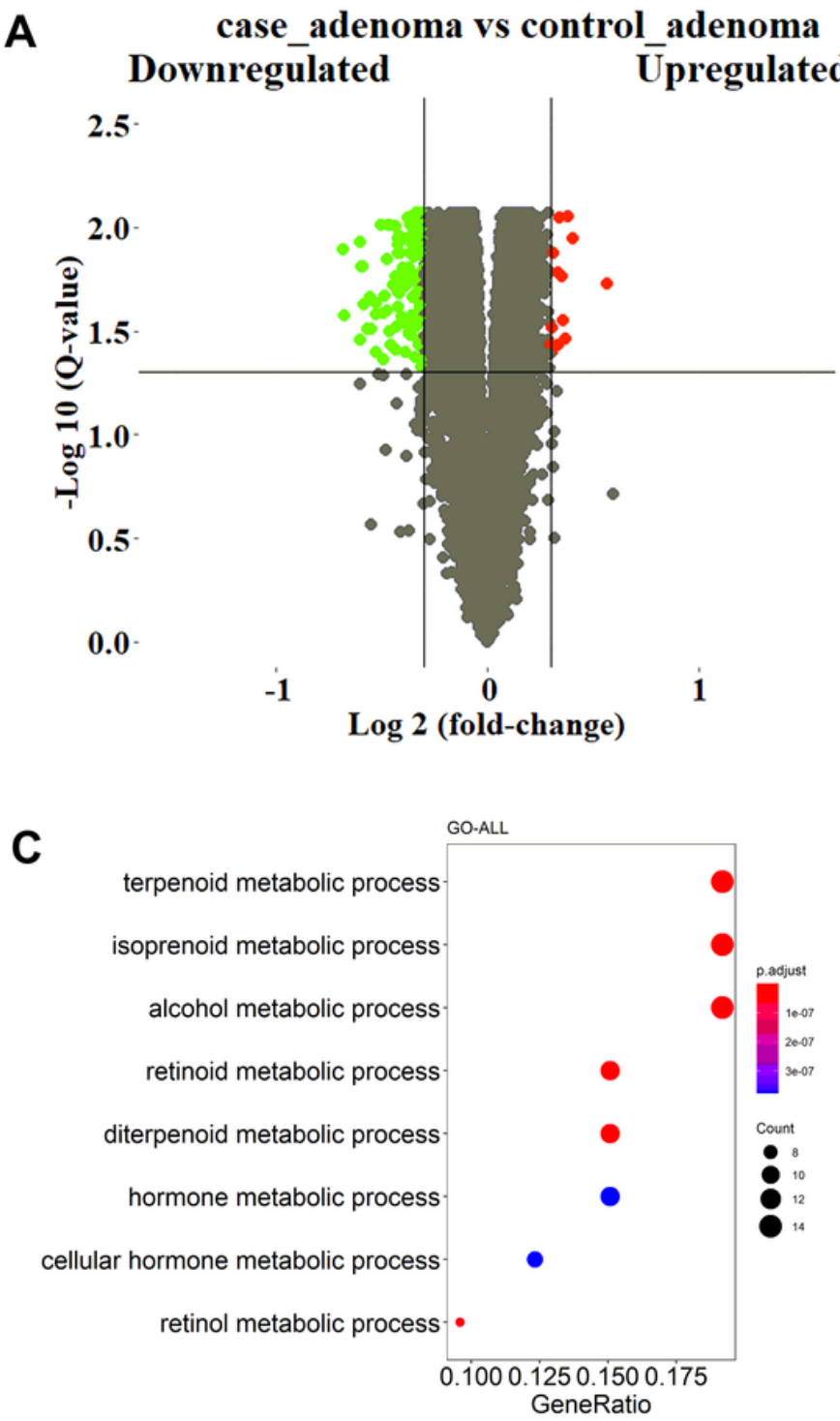

B

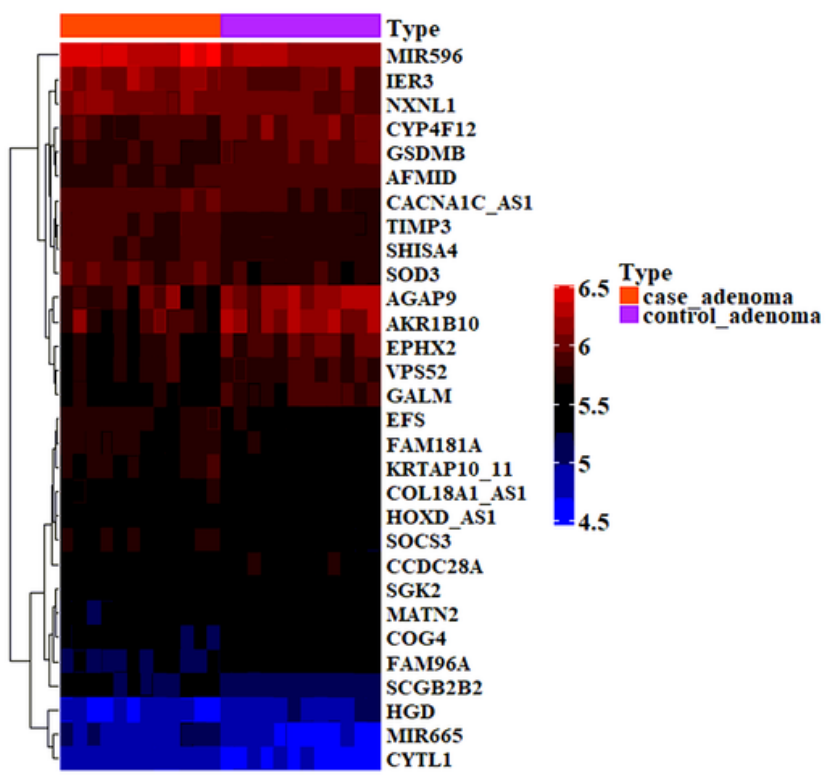

D

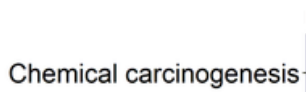

Drug metabolism - cytochrome P450 Retinol metabolism

Metabolism of xenobiotics by cytochrome P450

Fat digestion and absorption

Drug metabolism - other enzymes

Vitamin digestion and absorption. •

Fatty acid degradation -

0.0750 .1000 .1250 .1500 .175

GeneRatio

\section{Figure 2}

The differential expression analysis for adenoma tissue samples between FAP with and without duodenal carcinoma (case and control). (A) Volcano plot. (B) Heatmap depicted the top 30 DEGs. Dot plots of (C) GO and (D) KEGG enrichment. 

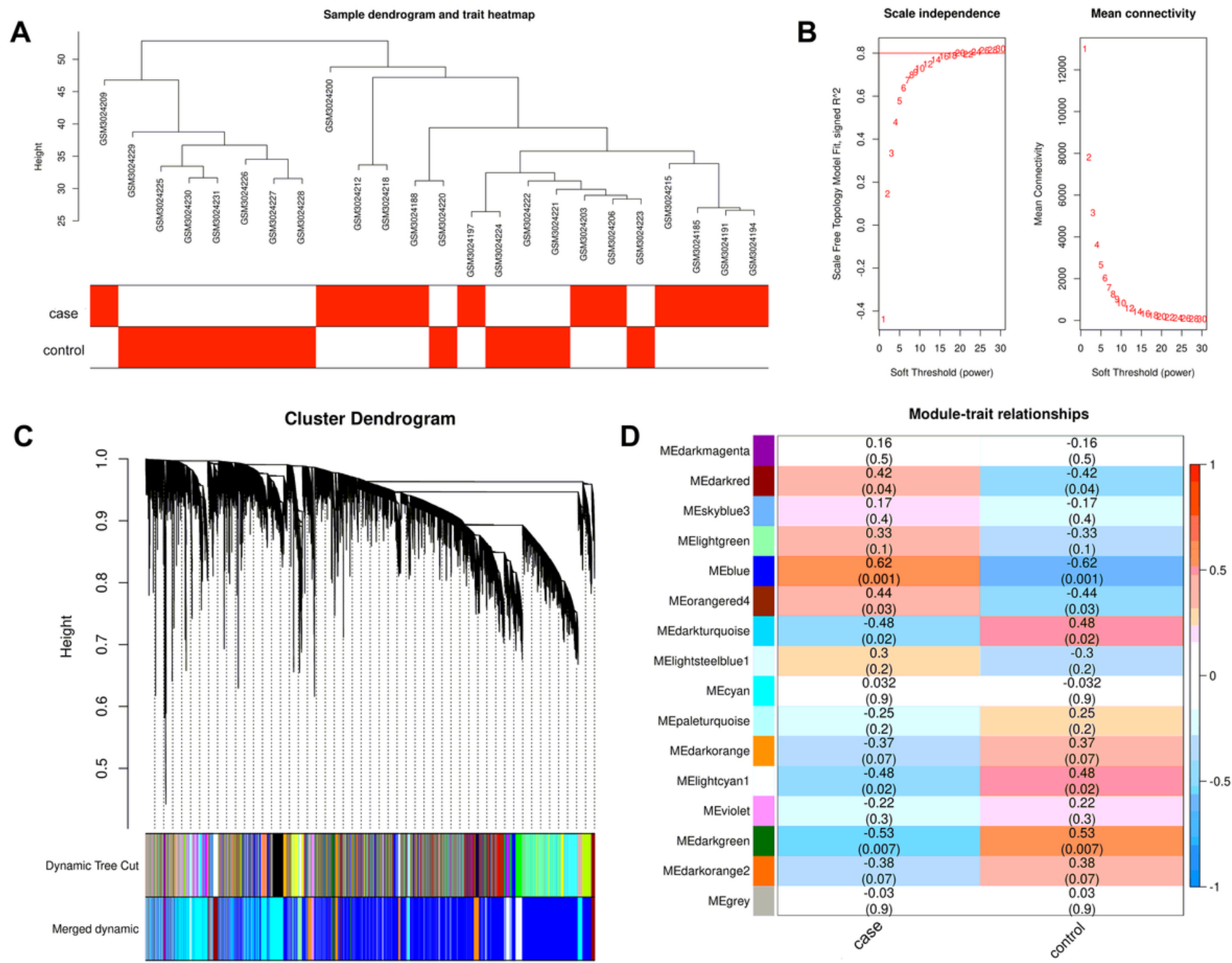

\section{Figure 3}

Weighted gene co-expression network analysis based on adenoma samples from case and control. (A) Sample dendrogram and trait heatmap. (B) Scale independence and mean connectivity of various softthresholding values ( $\beta$ ), $\beta=18$. (C) Dendrogram of all filtered genes enriched according to a dissimilarity measure (1-TOM) and the cluster module colors. (D) Heatmap of the correlation between the clinical traits and MEs of FAP. The darker the module color, the more significant their relationship. 
A

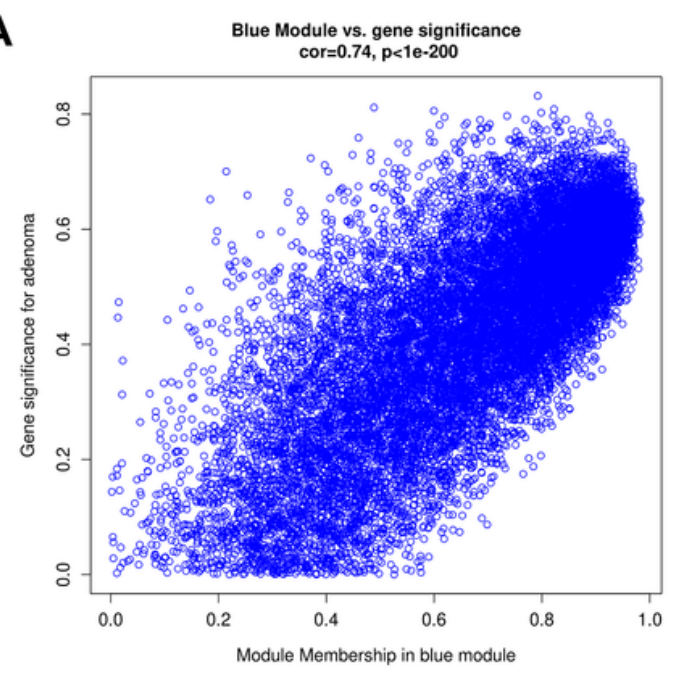

B

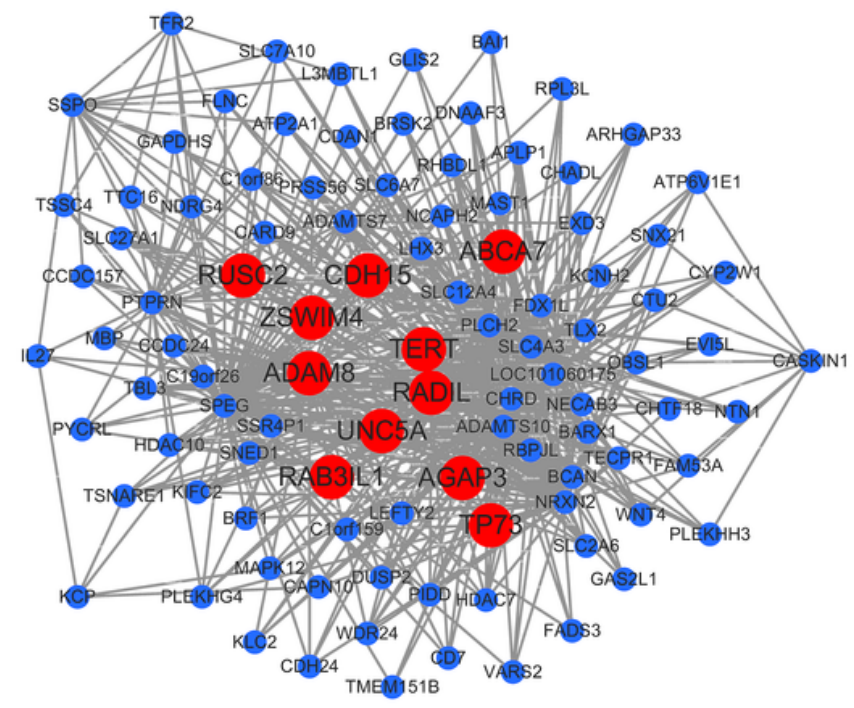

C

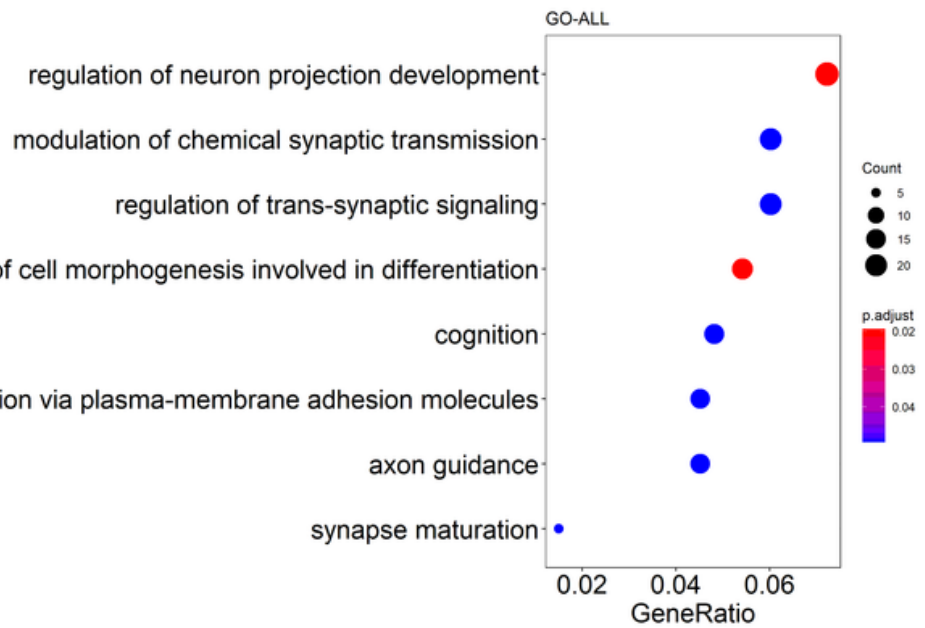

D

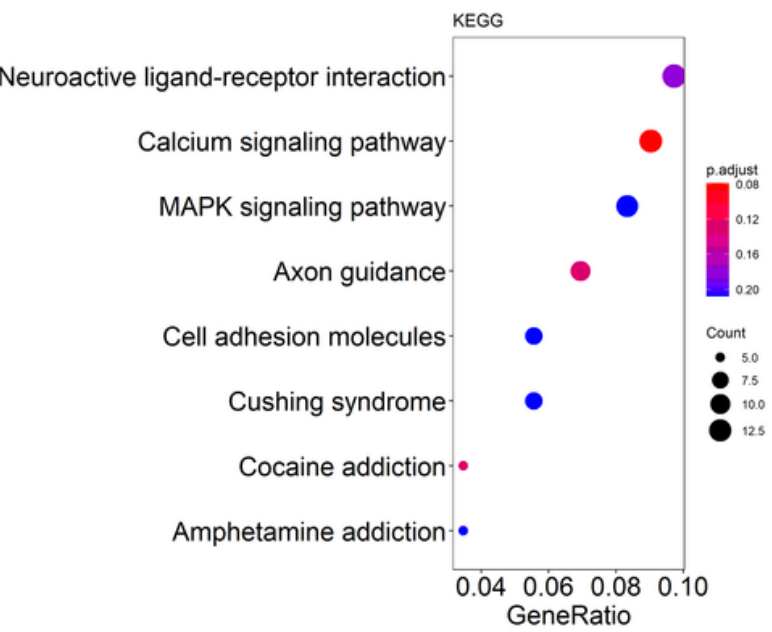

\section{Figure 4}

Analysis of the blue module associated with case. (A) The scatter plot between the blue module membership and the gene significance for case. (B) The gene-gene network containing 3 hub genes, suggesting the hub genes exhibited high connectivity with other genes. (C) GO and (D) KEGG enrichment results of genes in the blue module. 
A

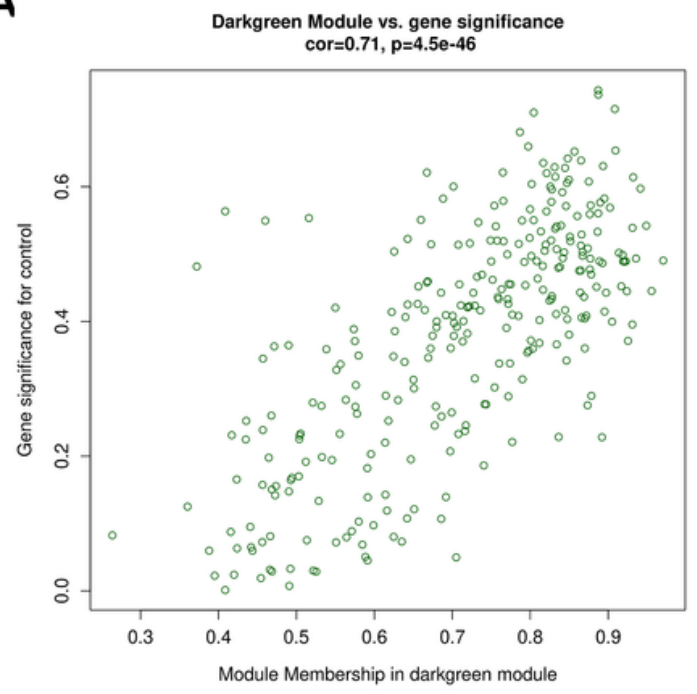

C

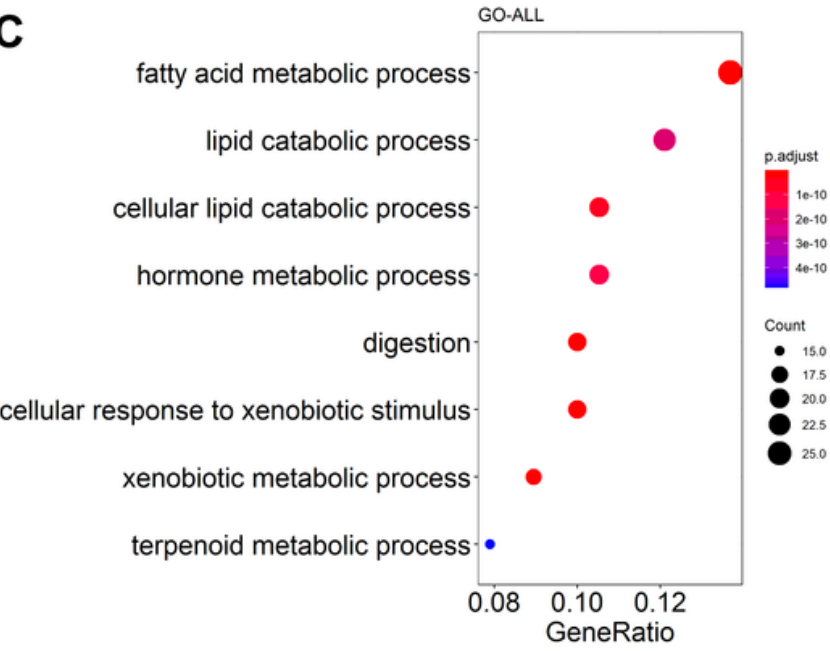

B
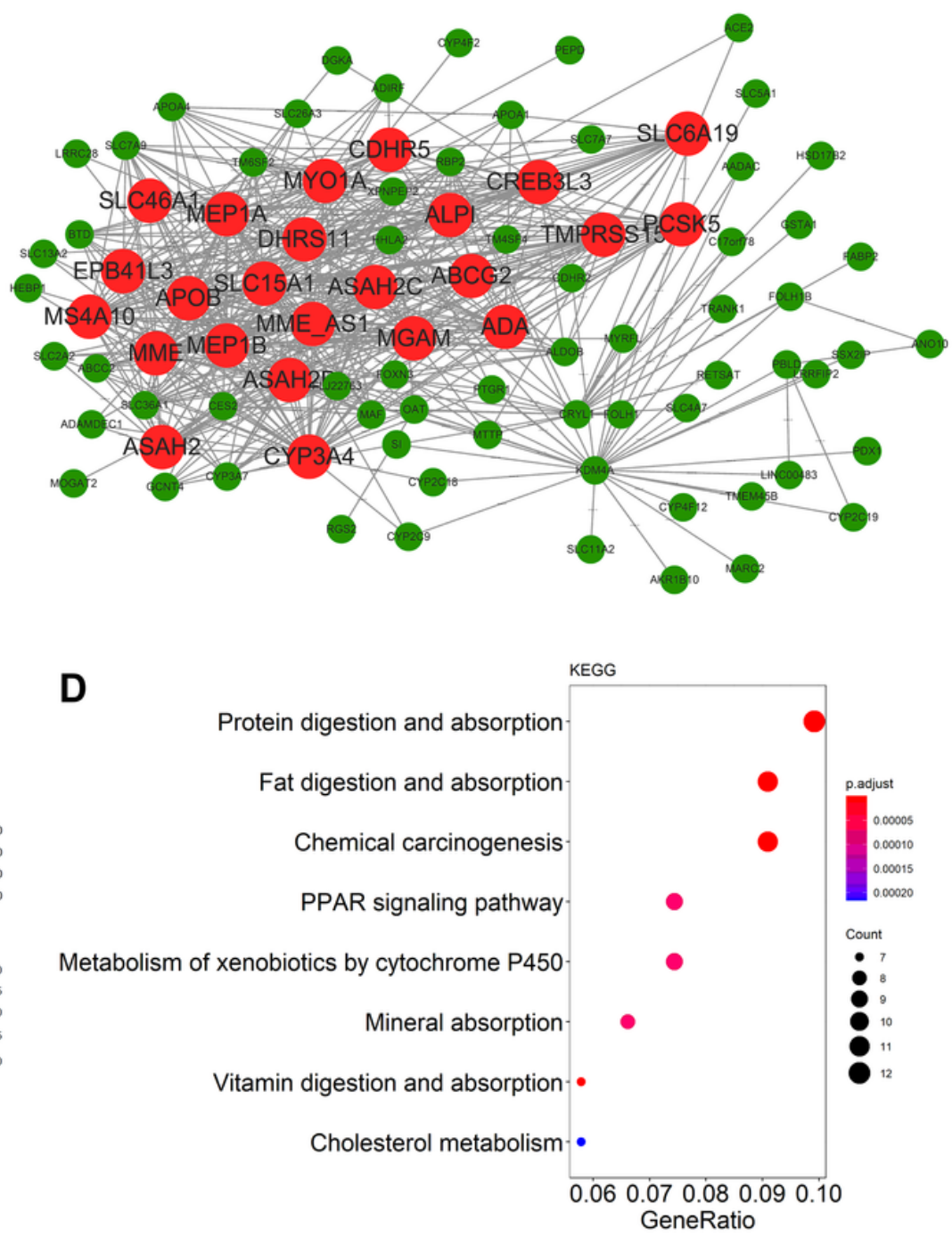

\section{Figure 5}

Analysis of the darkgreen module associated with control. (A) The scatter plot between the darkgreen module membership and the gene significance for control. (B) The gene-gene network containing 24 hub genes, suggesting the hub genes exhibited high connectivity with other genes. (C) GO and (D) KEGG enrichment results of genes in the blue module. 
A

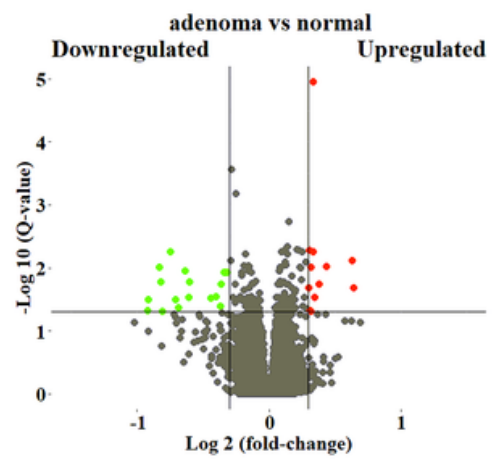

D

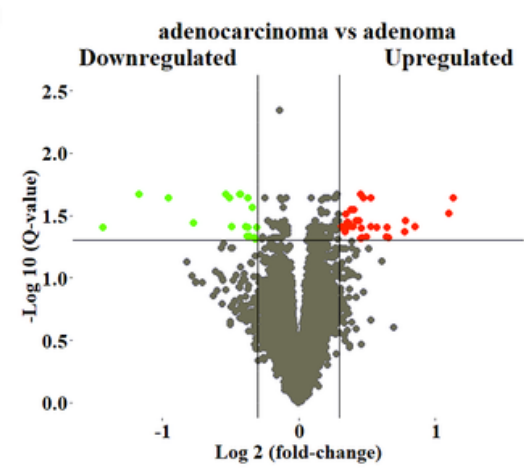

G

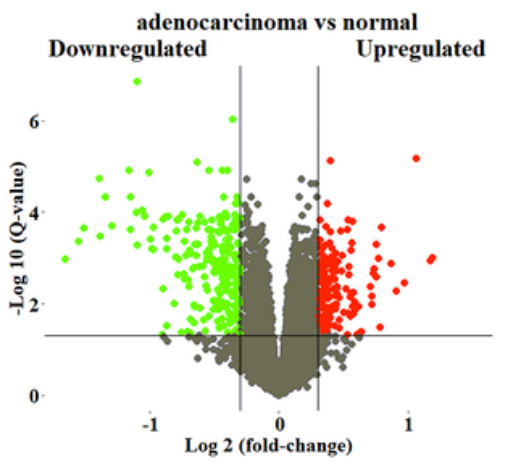

B

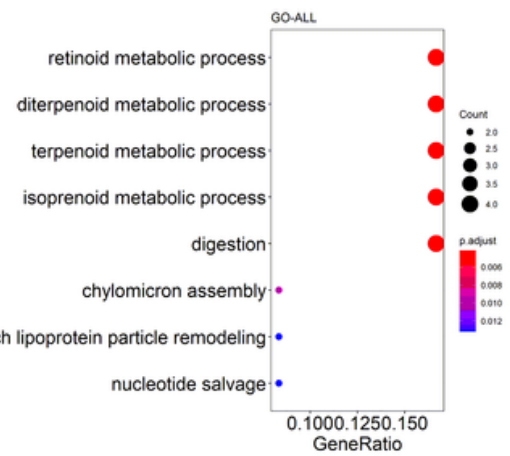

C

Chemin digeston and absorpton

Vitamin digestion and absorption

Protein digestion and absorption

Folate biosynthesis -

Fructose and mannose metabolism.

Fat digestion and absorption.

Cholesterol metabolism.

Glutathione metabolism.

triglyceride-rich lipoprotein particle remodeling-

Drug metabolism - cytochrome P450 •

0.100 .110 .120 .130 .140 .15
GeneRatio

E

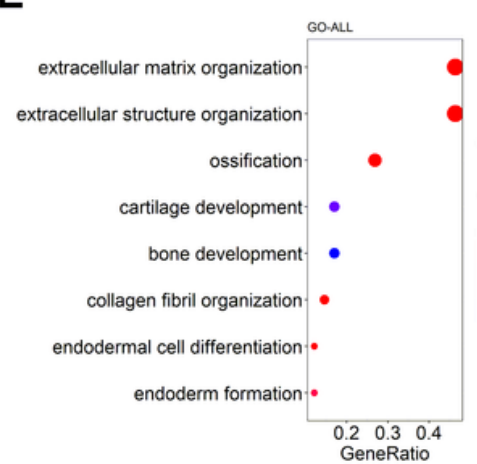

$\mathbf{F}$

H

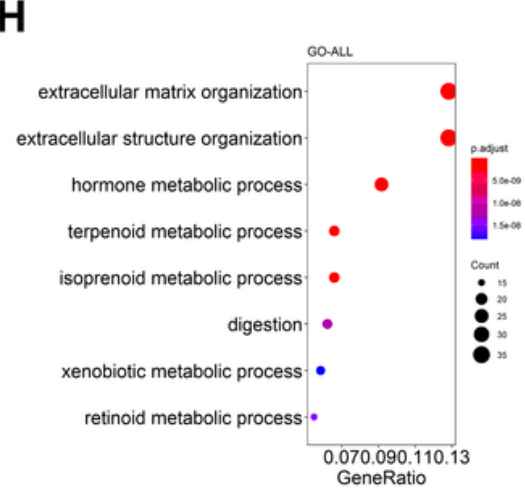

AGE-RAGE signaling pathway in diabetic complications

: Drug metabolism - cytochrome P450 -

Metabolism of xenobiotics by cytochrome P450 Chemical carcinogenes
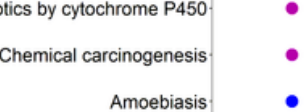

Tyrosine metabolism.

I

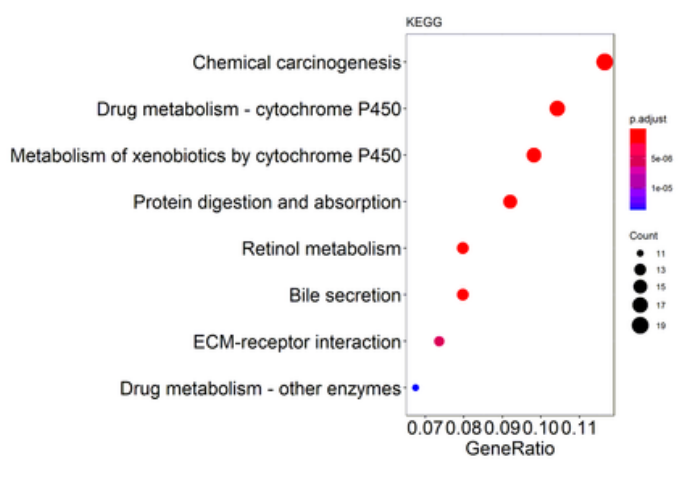

Figure 6

The differential expression analysis for different tissue samples (normal, adenoma, and adenocarcinoma) from FAP with duodenal carcinoma. (A) Volcano plot, (B) GO, and (C) KEGG enrichment dot plots of DEGs identified between normal and adenoma. (D) Volcano plot, (E) GO, and (F) KEGG enrichment dot plots of DEGs identified between adenocarcinoma and adenoma. (G) Volcano plot, $(H)$ GO, and (I) KEGG enrichment dot plots of DEGs identified between adenocarcinoma and normal. 
A

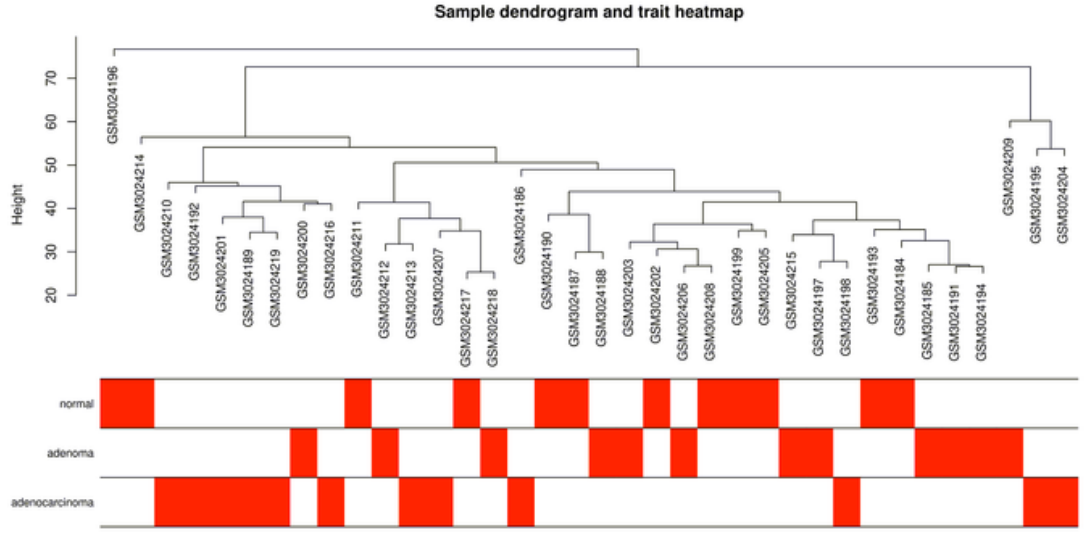

C

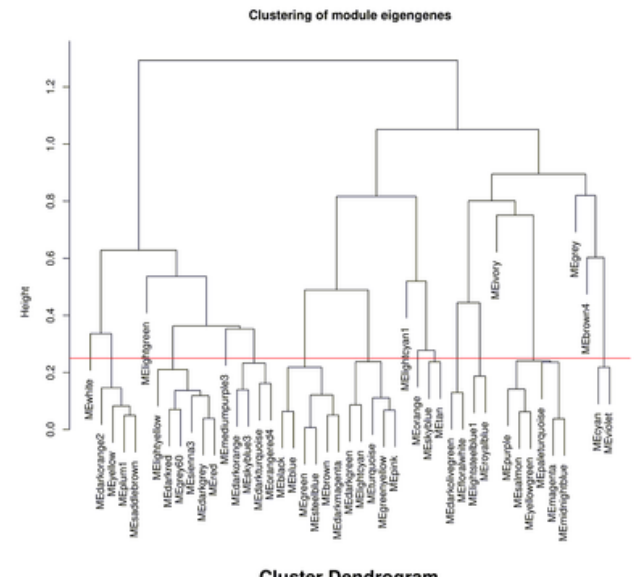

D

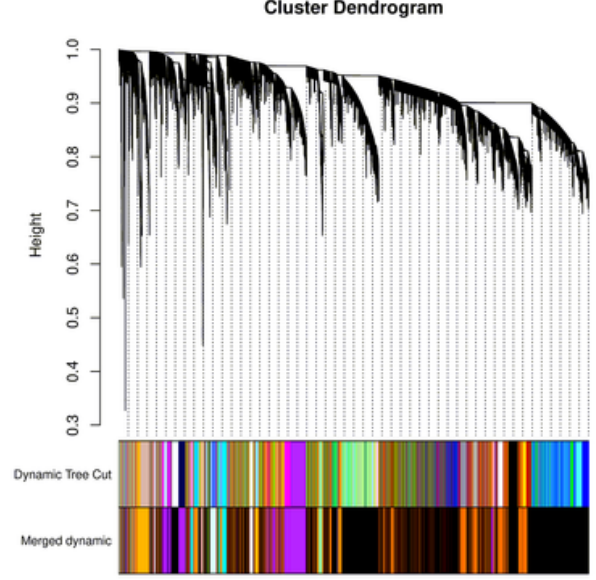

B

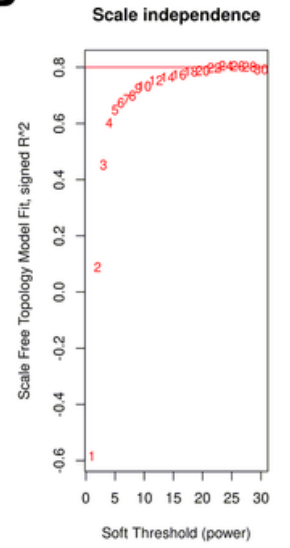

$\mathbf{E}$
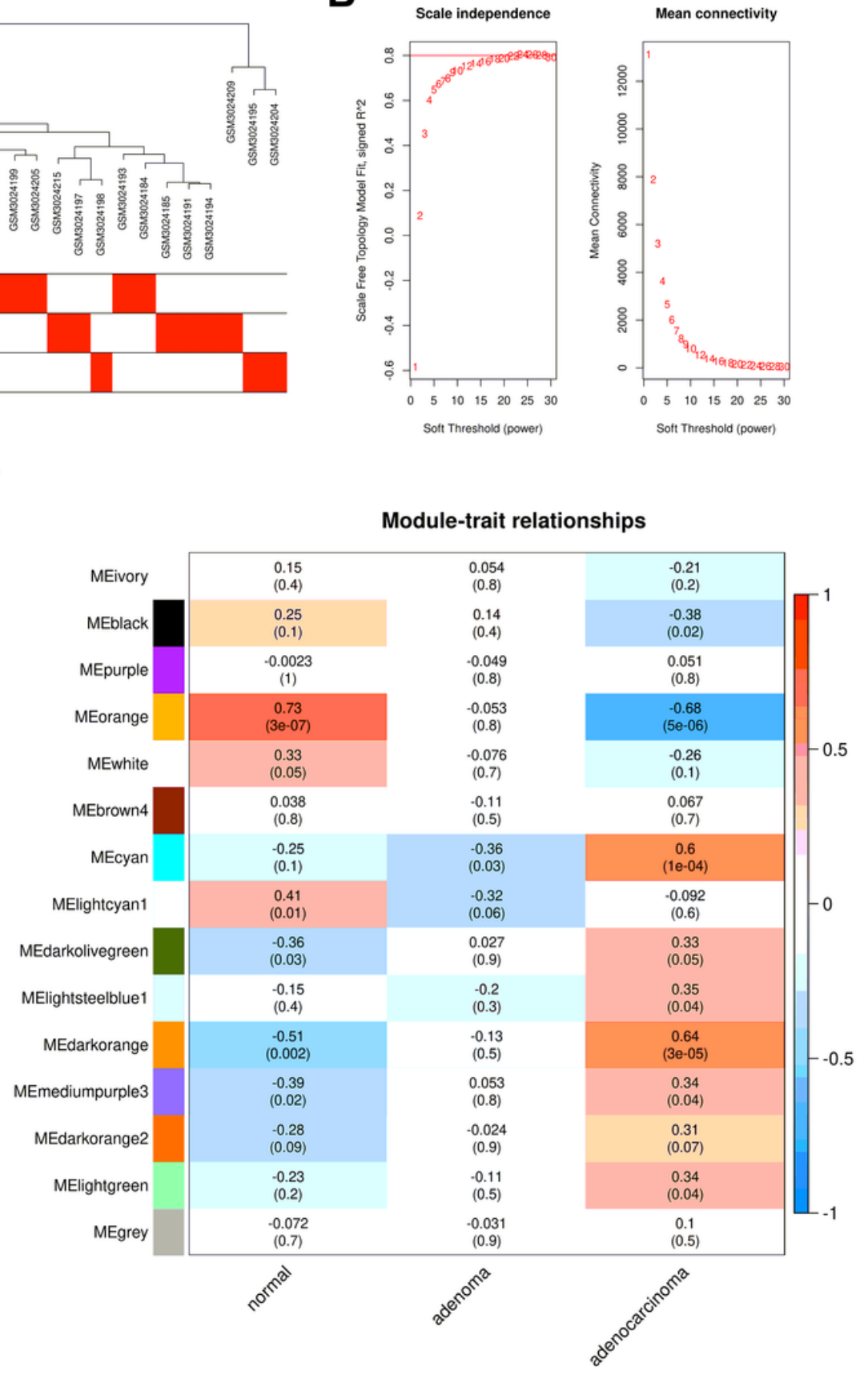

Figure 7

Weighted gene co-expression network analysis based on normal, adenoma, and adenocarcinoma samples from case. (A) Sample dendrogram and trait heatmap. (B) Scale independence and mean connectivity of various soft-thresholding values $(\beta), \beta=20$. (C) Dendrogram of consensus module eigengenes. The eigengenes groups were merged according to merging threshold (the red line) due to their similarity. (D) The cluster dendrogram of genes. (E) Heatmap of the correlation between the clinical traits and MEs of case. The darker the module color, the more significant their relationship. 
A

Orange Module vs. gene significance cor $=0.74, p=1.7 e-171$

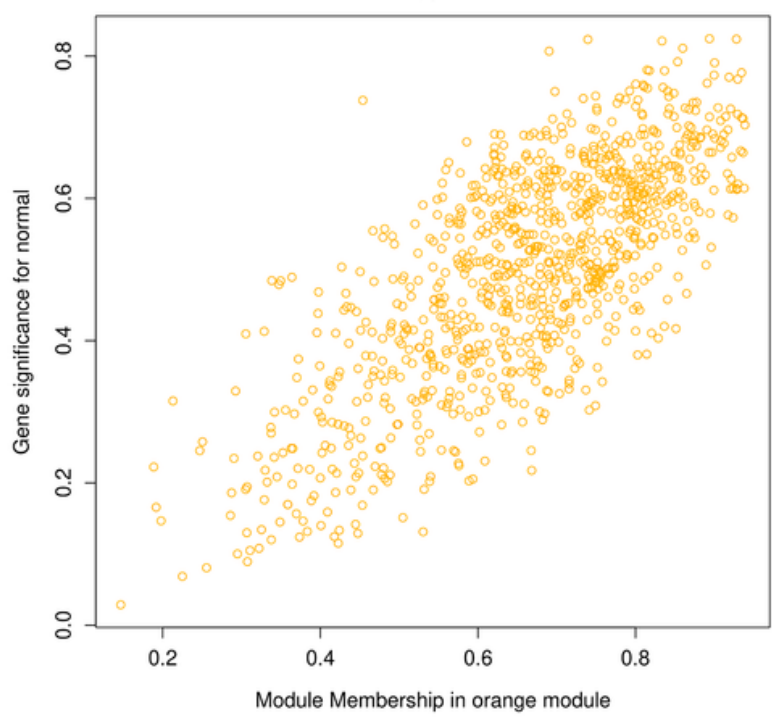

C

small molecule catabolic process fatty acid metabolic process lipid catabolic process cellular lipid catabolic process cellular response to xenobiotic stimulus

GO-ALL

\section{.}

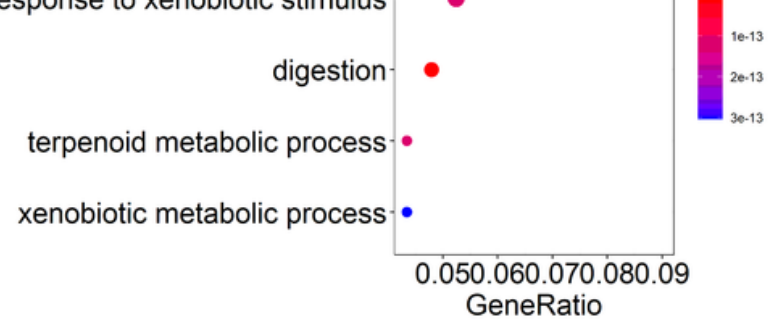

B

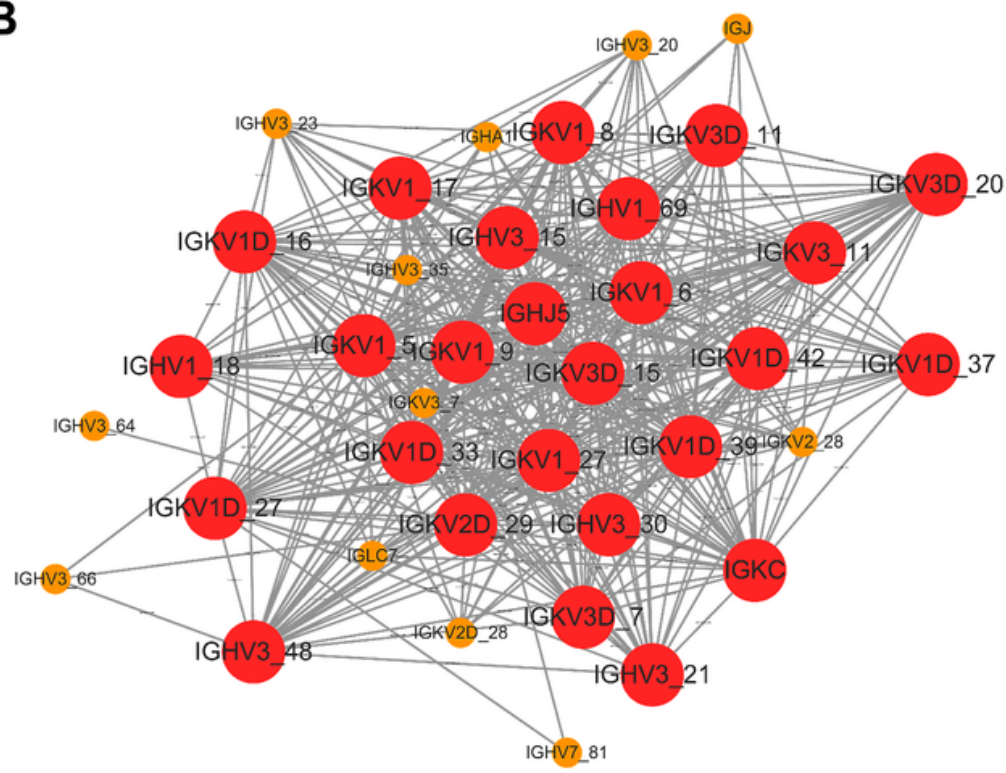

D

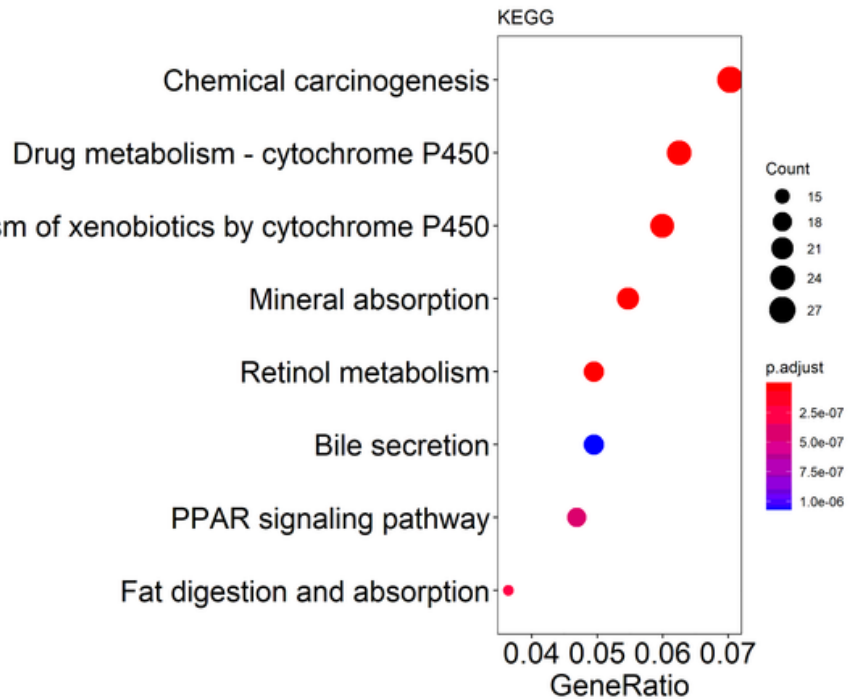

\section{Figure 8}

Analysis of the orange module associated with normal. (A) The scatter plot between the orange module membership and the gene significance for normal. (B) The gene-gene network containing 26 hub genes, suggesting the hub genes exhibited high connectivity with other genes. (C) GO and (D) KEGG enrichment results of genes in the orange module. 

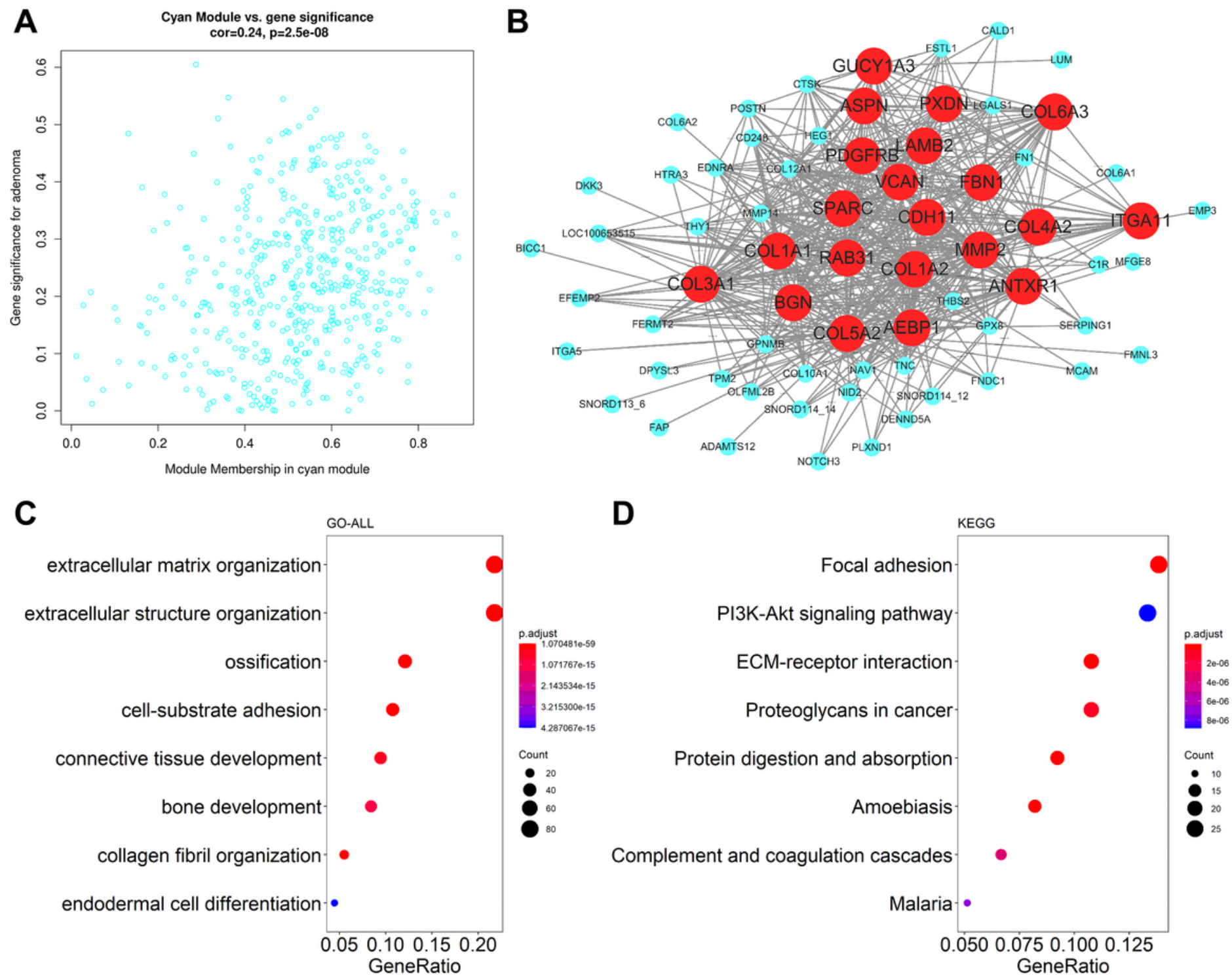

\section{Figure 9}

Analysis of the cyan module associated with adenoma. (A) The scatter plot between the blue module membership and the gene significance for adenoma. (B) The gene-gene network containing 21 hub genes, suggesting the hub genes exhibited high connectivity with other genes. (C) GO and (D) KEGG enrichment results of genes in the cyan module. 

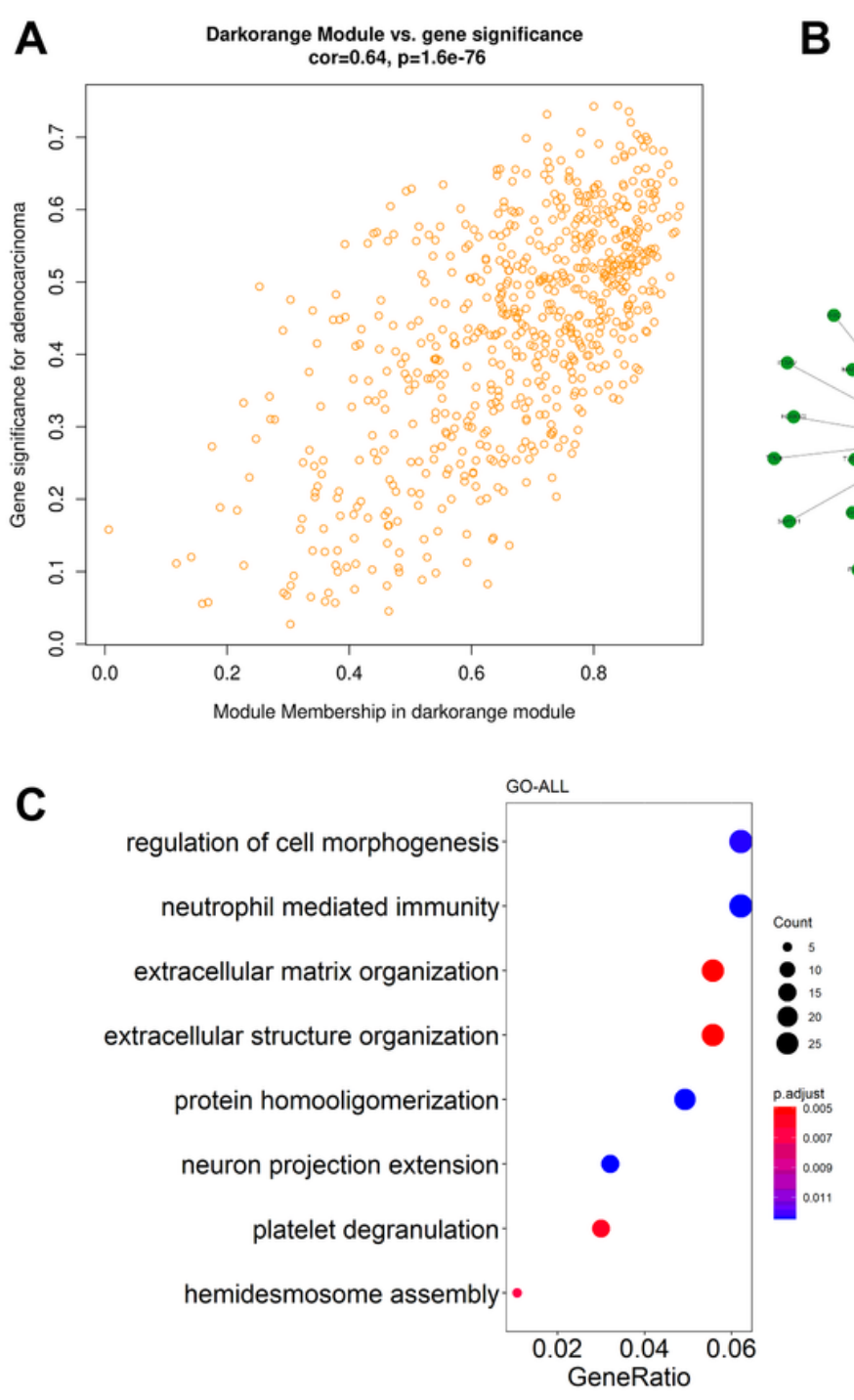

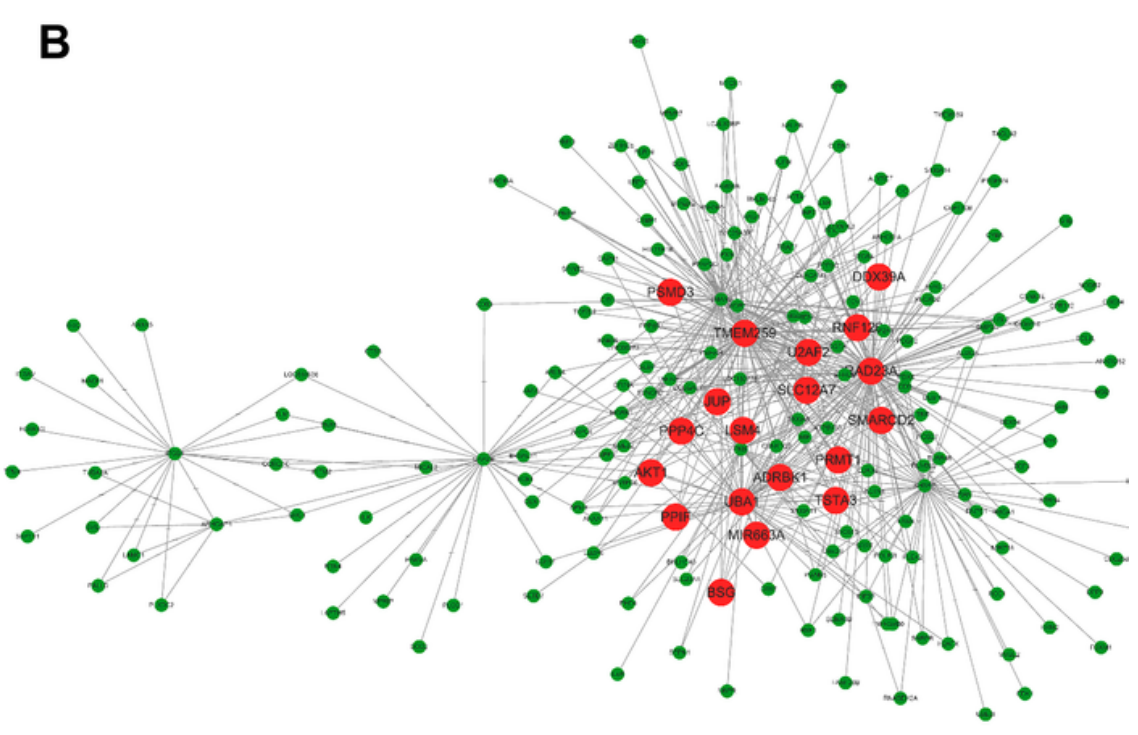

D

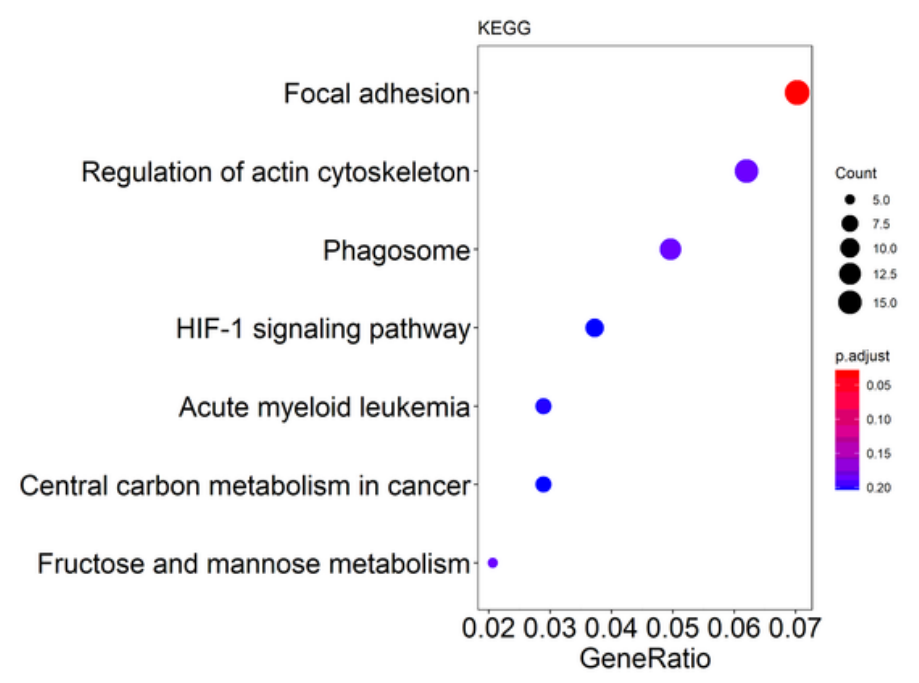

\section{Figure 10}

Analysis of the darkorange module associated with adenocarcinoma. (A) The scatter plot between the darkorange module membership and the gene significance for adenocarcinoma. (B) The gene-gene network containing 19 hub genes, suggesting the hub genes exhibited high connectivity with other genes. (C) GO and (D) KEGG enrichment results of genes in the darkorange module.

\section{Supplementary Files}

This is a list of supplementary files associated with this preprint. Click to download.

- FigureS1.tif

- TableS1.xlsx

- TableS2.xIsx

- TableS3.xIsx 
- Tables4.xlsx

- Tables5.xlsx

- TableS6.xlsx

- Tables7.xlsx 